\title{
Molecular Mechanism of Action of Mitochondrial Therapeutic SS-31 (Elamipretide): Membrane Interactions and Effects on Surface Electrostatics
}

Wayne Mitchell ${ }^{a}$, Emily A. Ng ${ }^{a}$, Jeffrey D. Tamucci ${ }^{a}$, Kevin Boyd ${ }^{a}$, Murugappan Sathappa ${ }^{b}$, Adrian Coscia ${ }^{c}$, Meixia Pan ${ }^{d}$, Xianlin $\operatorname{Han}^{\mathrm{d}, \mathrm{e}}$, Nicholas A. Eddy ${ }^{\dagger}$, Eric R. May ${ }^{\mathrm{a}}$, Hazel H. Szeto ${ }^{g}$, and Nathan N. Alder ${ }^{a, *}$

${ }^{a}$ Department of Molecular and Cell Biology, University of Connecticut, Storrs, CT 06269

${ }^{b}$ Present address: Center for the Development of Therapeutics, Broad Institute, Cambridge, MA, 02142

${ }^{\mathrm{C}}$ Present address: Department of Microbiology, Harvard Medical School, Boston, MA, 02115

${ }^{\mathrm{d} B a r s h o p}$ Institute for Longevity and Aging Studies, and ${ }^{\mathrm{e}}$ Department of Medicine, University of Texas Health Science Center at San Antonio, San Antonio, TX 78829

IInstitute of Materials Science, University of Connecticut, Storrs, CT 06269

${ }^{9}$ Social Profit Network Research Lab, Alexandria LaunchLabs, New York, NY, 10016

${ }^{*}$ Corresponding Author

Dr. Nathan N. Alder

91 N. Eagleville Rd

Storrs, CT 06269-3125

e-mail: nathan.alder@uconn.edu

phone: 860-486-5154

\section{Classification}

Major Category: Biological Sciences

Minor Category: Biophysics and Computational Biology

\section{Keywords}

Mitochondria; Peptide Therapeutic; Membrane; Bioenergetics; Cardiolipin 


\section{Significance}

Szeto-Schiller (SS) peptides are among the most promising therapeutic compounds for mitochondrial dysfunction. However, the molecular target(s) and the mechanism of action of SS peptides are poorly understood. In this study, we evaluate the interaction of the lead compound SS-31 (Elamipretide) with mitochondrial and synthetic model membranes using a host of biophysical techniques. Our results show that SS-31 membrane interaction is driven largely by the negative surface charge of mitochondrial membranes and that SS-31 alters lipid bilayer properties, most notably electrostatics at the membrane interface. This work supports a mechanism in which SS peptides act on a key physical property of mitochondrial membranes rather than with a specific protein complex, consistent with the exceptionally broad therapeutic efficacy of these compounds.

\section{Abstract}

Mitochondrial dysfunction includes heritable diseases, acquired pathologies, and age-related declines in health. Szeto-Schiller (SS) peptides comprise a class of amphipathic tetrapeptides that have demonstrated efficacy in treating a wide array of mitochondrial disorders, and are believed to target mitochondrial membranes due to their enrichment in the anionic phospholipid cardiolipin (CL). However, little is known regarding how SS peptides interact with or alter the physical properties of lipid bilayers. In this study, we have analyzed the interactions of the lead compound SS-31 (Elamipretide) with model and mitochondrial membranes using biophysical and computational approaches. Our results show that this polybasic peptide partitions into the membrane interfacial region with affinity and binding density that are directly related to surface charge. SS-31 binding does not destabilize lamellar bilayers even at the highest binding concentrations; however, it does cause saturable alterations in lipid packing. Most notably, SS31 modulates the surface electrostatic properties of model and mitochondrial membranes, which could play a significant role in the mitoprotective properties of this compound. As a proof of concept, we show that SS-31 alters ion distribution at the membrane interface with implications for maintaining mitochondrial membranes subject to divalent cation (calcium) stress. Taken together, these results support a mechanism of action in which SS peptides interact with lipid bilayers and alter the biophysical (primarily electrostatic) properties of mitochondrial membranes as their primary mechanism of action. Understanding this molecular mechanism is key to the development of future compound variants with enhanced efficacy. 


\section{Introduction}

Mitochondria are eukaryotic organelles that orchestrate the majority of cellular energy metabolism as well as a range of other processes including lipid biosynthesis, ion homeostasis, and programmed cell death (apoptosis). Mitochondrial dysfunction encompasses a wide array of both genetically encoded and acquired pathologies. Several mitochondrial diseases originate from heritable mutations in genes encoding mitochondrial proteins, either in nuclear or mitochondrial DNA (nDNA and mtDNA, respectively) (1). Moreover, reduced mitochondrial function accompanies the general decline in cellular bioenergetic capacity that occurs with aging as well as with many complex pathologies, including frailty, cardiomyopathy, cancer, and neurodegeneration (2). Features of mitochondrial dysfunction include gross alterations of the inner membrane cristae morphology, destabilization of respiratory complexes of the oxidative phosphorylation (OXPHOS) system, and overproduction of reactive oxygen and nitrogen species (ROS and RNS). Yet despite recent progress aimed at finding pharmacological approaches for mitochondrial disorders (3), there are presently no FDA-approved therapeutic compounds to treat them.

Szeto-Schiller (SS) peptides are among the most promising compounds currently under investigation for the treatment of mitochondrial dysfunction $(4,5)$. These synthetic tetrapeptides have a characteristic motif of alternating cationic and aromatic side chains (Fig. 1). Early in their development, SS peptides were shown to be cell-permeable in a wide range of cell types and to specifically target mitochondria. Despite their strong positive charge density (formal charge of +3 ), exogenously added SS peptides traverse the plasma membrane in an energy-independent and non-saturable manner, and accumulate strongly (1000 to 5000-fold) at the mitochondrial inner membrane $(6,7)$. The intrinsic therapeutic activity of the lead compound, SS-31 (Fig. 1A), was first demonstrated in cell culture studies, wherein the peptides were shown to protect cells against induced oxidative stress by curtailing oxidative cell death, reducing intracellular ROS, maintaining membrane potential $\left(\Delta \Psi_{\mathrm{m}}\right)$, and preventing lipid peroxidation, all in a dosedependent manner $(6,8)$. SS peptides have since been shown by many independent groups using cell culture and animal disease models to have significant efficacy in restoring mitochondrial function with a wide range of pathologies, including cardiomyopathy and heart failure, skeletal muscle injury/atrophy, ischemia and ischemia-reperfusion injury, kidney injury and disease, neurodegenerative diseases, cancer, and the heritable disease Friedreich's ataxia (summarized in ref. 5). These studies have all underscored the safety and favorable pharmacokinetic profile of SS peptides. Currently, Stealth BioTherapeutics is conducting early to 
advanced-phase clinical trials with SS-31 (proprietary name Elamipretide) for a range of primary mitochondrial and aging-related diseases.

Despite abundant evidence for the broad therapeutic potential of SS peptides, the molecular mechanism of action (MOA) of these compounds is poorly understood. It was originally proposed that SS peptides act as mitochondria-targeted antioxidants. Peptide variants such as SS-31, which contain a free radical-scavenging tyrosine (or dimethyltyrosine) moiety, could in principle serve to reduce ROS burden (9). Yet antioxidant chemistry is not likely to be the primary mechanism of SS peptides, given: (i) that such scavenging would not occur catalytically, and (ii) that other peptide variants such as SS-20 (Fig. 1B), which do not contain scavenging side chains, have also proven effective in preclinical studies.

Perhaps the most critical insight into the MOA of SS peptides is that they are believed to target the mitochondrial inner membrane (MIM) by virtue of its enrichment in the anionic phospholipid cardiolipin (CL) (10-13). CL has an unusual dimeric structure with a two-phosphate headgroup and four acyl chains (Fig. S1A) and comprises roughly $10-20$ mol\% of the total lipids within the MIM. CL plays a central role in many mitochondrial processes, including apoptosis, mitophagy, signaling, fission/fusion dynamics, and energy metabolism (14). Within the proteinrich MIM, CL mediates many interactions with peripheral and integral membrane proteins (15, 16), underpinning its role in the assembly of OXPHOS complexes into supercomplexes (17). Moreover, with its unique physicochemical properties and structural polymorphism, CL has a complex phase behavior in lipid bilayers $(18,19)$. Thus, local enrichment of CL can promote negative curvature, which likely helps to stabilize cristae architecture $(20,21)$. Nascent $C L$ is synthesized de novo within the MIM and subsequently undergoes a remodeling process to render mature species with a highly unsaturated complement of acyl chains that is species- and tissue-specific (22) (Fig. S1B). Alterations in CL distribution and biogenesis are associated with a number of diseases (23), including Barth syndrome (24), which is caused by defects in the transacylase tafazzin with concurrent aberrant remodeling of $\mathrm{CL}$ and buildup of the its lysolipid form, monolysocardiolipin (MLCL). Hence, being critical for mitochondrial structure and function, the anionic phospholipids of mitochondrial membranes are promising targets for therapeutic agents.

A primary mode of interaction between SS peptides and CL-rich lipid bilayers would be a truly unparalleled type of MOA. This is because the vast majority of drug compounds are designed to target and act upon specific proteins, not the lipid bilayer (with rare exceptions such as general anesthetics, whose mechanisms may involve alteration of bilayer properties (25)). In this study, we analyzed the nature of the SS-31 interaction with biomimetic model membranes 
and intact mitochondria to understand the forces that drive the peptide-membrane interaction and how SS-31 binding affects physical properties of model and natural lipid bilayers. Our results support a molecular MOA that involves alteration of bulk lipid bilayer properties, most notably the membrane surface electrostatic profile. We discuss multiple nonexclusive mechanisms by which the regulated tuning of surface electrostatics could underpin the broad therapeutic efficacy of SS peptides.

\section{Results}

\section{Binding of SS-31 to model membranes}

The interaction of basic amphipathic peptides with negatively charged membranes depends on the electric field originating from the bilayer surface as well as specific electrostatic and hydrophobic interactions between the peptide and lipids (Fig. S2). The first objective of this study was to quantitatively evaluate the binding of SS-31 to model membranes with specific lipid composition. For spectroscopic analysis of SS-31 binding, we made use of the endogenous spectral properties of the $2^{\prime}, 6^{\prime}$-dimethyltyrosine $\left(2^{\prime}, 6^{\prime}-\mathrm{Dmt}\right)$ side chain at the second position in the peptide. Our initial spectral characterization of SS-31 confirmed the feasibility of using endogenous peptide fluorescence to quantify peptide-membrane interactions (SI Results A,B and Figs. S3, S4). We therefore proceeded to measure the binding of SS-31 to large unilamellar vesicles (LUVs) containing anionic lipids in a host background of the zwitterionic lipid palmitoyloleoyl-phosphatidylcholine (POPC, 16:0/18:1 PC). The anionic lipids included tetraoleoylcardiolipin (TOCL, all acyl chains 18:1), monolysocardiolipin (MLCL, all acyl chains 18:1), or palmitoleoyl-phosphatidylglycerol (POPG, 16:0/18:1 PG). Binding measurements were performed using two complementary approaches: (i) titration of fixed concentrations of LUVs with peptide, and (ii) titration of fixed concentrations of peptide with LUVs (SI Results C and Fig. S5). Note that in our analyses, we assume that peptide does not cross the bilayer of our model systems and can only access the outer leaflet; for LUVs of this size, the 'effective lipid concentration' of the outer leaflet $\left([L]^{\text {eff }}\right)$ is taken as half of the total lipid concentration in the system (26).

Binding isotherms based on SS-31 emission intensity are shown in Fig. 2. First, peptide titrations were conducted by the progressive addition of SS-31 to LUVs of different composition at lipid concentrations ranging from 25-125 $\mu \mathrm{M}$ (Fig. 2A, see also SI Results D and Figs. S6 and S7). Second, lipid titrations were conducted by the progressive addition of LUVs to SS-31 at concentrations up to $15 \mu \mathrm{M}$ (Fig. 2B). Taken together, these binding curves reveal the following: (i) in the absence of anionic lipid (LUVs with POPC only), SS-31 binding is negligible 
even at the highest lipid concentrations used, consistent with the established requirement of anionic lipids for binding (10-13); (ii) in the presence of anionic membranes, SS-31 binding displays saturation binding behavior; and (iii) membranes containing dianionic lipid (TOCL and MLCL) have a higher SS-31 binding capacity than membranes containing monoanionic POPG, reflected in the roughly twofold higher [SS-31] required to saturate binding in peptide titrations and in the roughly twofold lower [lipid] required to saturate binding in lipid titrations.

To obtain equilibrium binding parameters $n$ (the number of lipid molecules per peptide bound) and $K_{\mathrm{D}}$ (the dissociation constant), we analyzed binding isotherm data by Scatchard analysis (Fig. 2C) and by fits of the data to Langmuir adsorption models (Eq. S2 and Eq. S3 for peptide and lipid titrations, respectively). The equilibrium binding parameters from this work (Fig. 2E, left panels) reveal two key points. First, for membranes containing cardiolipin variants, the average lipid:peptide stoichiometry ( $n=6.9$ and 7.4 for TOCL and MLCL, respectively) is roughly half the value for membranes with POPG $(n=15.4)$. Hence, if we assume ideal lipid mixing and approximate lipid cross-sectional areas $\left(70 \AA^{2}\right.$ for POPC and POPG; $130 \AA^{2}$ for TOCL and $\left.110 \AA^{2} \operatorname{MLCL}(27,28)\right)$, then for membranes composed of $20 \%$ TOCL or MLCL, the SS-31 "binding site" comprises an area of $\sim 560-580 \AA^{2}$ in which each peptide associates, on average, with 1.4-1.5 dianionic lipids. By comparison, for membranes containing 20\% POPG, SS-31 binds an area of roughly $1080 \AA^{2}$ and associates on average with 3 monoanionic lipids. Based on these stoichiometric relationships, then, there is an approximate charge balance between each peptide and its corresponding lipid binding site. Second, the affinity of SS-31 for membranes containing TOCL and MLCL is significantly higher than it is for membranes containing POPG. Specifically, the average dissociation constants for lipid monomers (reflected in $K_{\mathrm{D}}$ values for lipid titrations and in $n K_{\mathrm{D}}$ values for peptide titrations) are 2.0-2.9 $\mu \mathrm{M}$ for membranes composed of $20 \%$ TOCL and MLCL, whereas they range from 6.0-13 $\mu \mathrm{M}$ for membranes composed of 20\% POPG. These values of $n$ and $K_{\mathrm{D}}$ are consistent with parameters measured for other amphipathic membrane-active peptides $(29,30)$. As an independent measure of peptide binding, the molar partition coefficients $\left(K_{\mathrm{P}}\right.$, Eq. S9) for membranes containing $20 \%$ TOCL $\left(K_{P}=5.07 \pm 0.76 \times 10^{4}\right)$ and $20 \% \mathrm{MLCL}\left(K_{\mathrm{P}}=5.90 \pm 0.44 \times 10^{4}\right)$ are significantly higher than that of $20 \%$ POPG-containing membranes $\left(K_{P}=1.28 \pm 0.63 \times 10^{4}\right)$.

As a complementary approach for measuring SS-31 membrane interactions, we performed isothermal titration calorimetry (ITC) measurements by the progressive injection of LUVs into solutions of peptide in the sample cell (31). The interaction of SS-31 with membranes containing anionic lipid was shown to be exothermic in all cases (Fig. S8), and fits of complete binding isotherms (Fig. 2D) yielded the thermodynamic binding parameters shown (Fig. 2E, 
right panels). The values of $n$ and $K_{D}$ from fits to the ITC data showed consistent differences between TOCL/MLCL- and POPG-containing membranes when compared with our spectralbased measurements (Fig. 2). However, in comparison with our spectral analyses, there were differences in the absolute values from ITC-derived parameters (mean $n$ values slightly lower and $K_{\mathrm{D}}$ values $\sim 2-3$ times higher). This difference could be related to different phenomena being measured: our spectral analyses may only reflect the binding event per se, whereas ITC measurements could be reflecting binding as well as peptide-dependent alterations of bilayer properties. Apropos of this point, we did observe a slight asymmetry in our exothermic peaks, particularly for TOCL- and MLCL-containing membranes, which could be related to thermotropic alterations in the bilayers following peptide interaction.

Comparison of the binding-associated changes in enthalpy $(\Delta H)$ and entropy $(\Delta S)$ provide insights into the membrane binding mechanism. Namely, exothermic $(\Delta H<0)$ values of peptide-lipid interaction originate mainly from the establishment of polar contacts; by contrast, increases in the system entropy $(\Delta S>0)$ originate mainly from the burial of hydrophobic side chains in the acyl chain region with concurrent release of water and ions from nonpolar surfaces of the peptide and bilayer (32). For membranes containing TOCL, MLCL, and POPG, the interaction of SS-31 was associated with a favorable enthalpic change $(\Delta H=-4.2,-4.7$, and -2.1 kJ mol ${ }^{-1}$, respectively); the twofold difference in $\Delta H$ between TOCL/MLCL- and POPGcontaining membranes is consistent with the difference in formal charges of these anionic lipids. The peptide-bilayer association, however, was predominantly entropy-driven $(-T \Delta S=-22.7$, 20.8 , and $-21.5 \mathrm{~kJ} \mathrm{~mol}^{-1}$, respectively), showing that peptide association is stabilized mostly by burial of the nonpolar residues. Given that the hydrophobic contribution to peptide binding energy is proportional to the exposed side chain area $\left(\sim 80 \mathrm{~J} \mathrm{~mol}^{-1} \AA^{-2}\right)$ (33) the burial of Tyr (similar to $2^{\prime}, 6^{\prime}$-Dmt) and Phe side chains (accessible surface areas 187 and $175 \AA^{2}$, respectively) would theoretically contribute a combined $\sim 28 \mathrm{~kJ} \mathrm{~mol}^{-1}$, in reasonable agreement with our measurements. Moreover, because SS peptides are short and cannot form any appreciable secondary structure upon membrane binding, their membrane binding does not likely incur an entropic penalty that would be associated with stabilizing a secondary structure.

Taken together, our spectral and calorimetric binding data show that the bilayer binding density and affinity of SS-31 are directly related to the anionic lipid composition, that the lipiddependent differences in interaction energy are mostly on the net charge of anionic lipids, and that membrane interactions are enthalpically and entropically favorable.

\section{Dependence of SS-31 binding on model membrane surface electrostatics}


The electrostatic profile of biomembranes consists of multiple potential energy functions (Fig. S2). Among them, the surface potential $\left(\psi_{s}\right)$ originates from charge distribution at the membrane interface based on ionizable functional groups (e.g., lipid headgroups) and adsorbed ions, creating a strong electrostatic driving force for the binding of charged moieties to the bilayer surface (34). Electrolytes partitioned at the bilayer interface can have a complex effect on membrane interactions of amphipathic peptides. By attenuating the $\psi_{\mathrm{s}}$, solution cations can reduce the electrostatic attraction of basic peptides to the anionic lipid surface; however, increasing solution ionic strength may also favor the burial of hydrophobic side chains in the nonpolar core of the bilayer. We therefore explored the relationship between SS-31 binding and the $\psi_{\mathrm{s}}$ of model membranes and the influence of ionic strength (Fig. 3).

SS-31 binding curves measured by titration of SS-31 with LUVs containing $20 \%$ TOCL or POPG revealed a strong effect of monovalent electrolyte (Fig. 3A). We confirmed that SS-31 binding to model bilayers was reversible in a manner that is dependent upon solution ionic strength used for these binding isotherms (SI Results E and Fig. S9A,B). When binding parameters $n$ and $K_{D}$ calculated from the curves of Fig. $3 A$ are considered as a function of salt concentration, there is a large disparity between membranes containing divalent TOCL and monovalent POPG (Fig. 3B, upper panels). However, recasting these parameters as a function of $\psi_{\mathrm{s}}$, calculated from Gouy-Chapman-Stern (GCS) formalism $(35,36)$ (Eq. S10-S12), reveals a much smaller difference between membranes containing the two anionic lipids (Fig. 3B, lower pane/s). This GCS analysis, which accounts for the roughly twofold larger intrinsic surface charge $\left(\sigma^{\max }\right)$ of TOCL- vs. POPG-containing bilayers, indicates that SS-31 binding is more related to $\psi_{\mathrm{s}}$ per se, rather than to any specific features of CL.

To continue this analysis, we determined the relationship between SS-31 binding and surface electrostatics by two complementary approaches. We first performed zeta potential $(\zeta)$ measurements of LUVs containing 20 mol\% TOCL or POPG with increasing [SS-31] (Fig. 3C). The $\zeta$ represents the electrostatic potential at the hydrodynamic shear plane of membranes, and is related to the $\psi_{s}$ by the Debye constant $(\kappa)$, which describes the position-dependent decay of electrostatic potential from the membrane surface to the bulk solution. As expected for the binding of a polybasic peptide to a negatively charged surface, SS-31 caused a saturable reduction in the magnitude of $\zeta$ for both anionic bilayers. As an independent technique, we measured the effect of SS-31 on surface electrostatics using 1,8-ANS, a fluorescent reporter whose binding to anionic bilayers increases as the magnitude of the $\psi_{\mathrm{s}}$ is reduced, measured as an increase in probe emission intensity (37) (Fig. 3D and Fig. S9C). Consistent with our $\zeta$ data, 
SS-31 caused a saturable 1,8-ANS-detected reduction in the $\psi_{\mathrm{s}}$ of anionic model membranes. We observed the same response with SS-20 (Fig. S9D), supporting that this effect on membrane surface electrostatics is a general feature of SS peptides. Beyond the observation that SS peptides attenuate membrane surface potential, two features are notable from these experiments. First, for both $\zeta$ and 1,8-ANS measurements, SS-31 elicited a hyperbolic decay of $\psi_{\mathrm{s}}$ for PG-containing bilayers, whereas the response was more sigmoidal for CL-containing bilayers (Fig. S9C,D blue vs. red). This suggests that at low peptide concentration, there is not a linear correspondence between peptide binding and charge attenuation for CL-containing membranes. Second, the $\zeta$ responses, which provide an absolute measure of $\psi_{\mathrm{s}}$, consistently saturated near $-30 \mathrm{mV}$ with increasing peptide. This indicates that when the model bilayers are maximally bound with peptide, there remains an appreciable negative surface charge density. This observation is in contrast to other basic peptides (38) and multivalent cations (39) that cause charge overcompensation (lead to positive surface charge density) upon binding anionic bilayers at high concentration. Two nonexclusive explanations may account for this: (i) SS-31 binding may render some ionized phosphate groups unavailable for further interaction, and/or (ii) the formal charge on SS-31 may become reduced upon binding. Regardless of the mechanism, the fact that SS-31 attenuates the $\psi_{\mathrm{s}}$, but does not completely reverse it, is a fundamental feature of its effect on the membrane surface electrostatic profile.

\section{SS-31 maintains the lamellarity of model membranes but alters lipid interactions at the interface}

Amphipathic molecules such as antimicrobial peptides can induce large structural changes in membranes, including micellization, pore formation, and induction of inverted topologies (40). We therefore addressed whether SS-31 induced structural polymorphism within model bilayers using a range of approaches (Fig. 4). Based on synchrotron small angle x-ray scattering (SAXS) measurements, LUVs composed of POPC alone or with $20 \%$ TOCL, MLCL, or POPG yielded scattering profiles typical of lipid vesicles (41), and the presence of SS-31 did not cause structural perturbation (Fig. 4A, SI Results F, and Fig. S10A). Similarly, based on ${ }^{31} \mathrm{P}$ solid state NMR (ssNMR) measurements, these model membranes yielded lineshapes consistent with lamellar bilayers (42), and we observed no effect of SS-31 on bilayer structure (Fig. 4B, SI Results F, and Fig. S10B). We conclude that even at the highest peptide concentration used in this study $\left([\mathrm{P}]:[L]^{\text {eff }}=1: 5\right)$, SS-31 did not cause major topological alterations of our model membranes. Thus, these membranes are expected to exist stably in the liquid crystalline lamellar mesophase over all peptide concentrations. 
We therefore addressed whether SS-31 altered lipid interactions within these model bilayers. To this end, we used membrane-bound fluorescent reporters of lipid dynamics and packing that partition into the bilayer at different depths (Fig. S10C). Using DPH anisotropy $\left(<r>^{\mathrm{DPH}}\right)$ as a readout of the fluid dynamics of hydrocarbon tails, we observed no effect of SS-31 in TOCL- or POPG-containing membranes (Figs. S10D,E). We then used solvatochromic probes laurdan and prodan, whose spectral properties are quantified as the generalized polarization ( $G P^{L A U}$ and $G P^{P R O}$, respectively) that increases with enhanced lipid packing (reduced interfacial hydration) (43) (SI Results F and Fig S10F). We observed modest, yet repeatable and saturable increases in $G P^{L A U}$ and $G P^{P R O}$ that corresponded to the fractional occupancy of peptide on the membrane (Fig. 4C). Notably, the response of GP values in TOCLcontaining bilayers was much higher in magnitude compared to the response in those with POPG. Based on these results, SS-31 binding causes a change in the hydration/polarity of the bilayer interface that likely results from an increase in lipid headgroup packing density.

\section{Molecular dynamics analysis of the interaction between SS-31 and lipid bilayers}

We next used all-atom molecular dynamics (MD) simulations to analyze the interaction between SS-31 and lipid bilayers (Fig. 5, SI Results G, and Figs. S11-S14). These simulation systems contained solvated bilayers composed of $20 \mathrm{~mol} \%$ TOCL, MLCL, or POPG in a host background of POPC, and were conducted in the presence or absence of SS-31 to evaluate SS-31-bilayer interactions and how the presence of SS-31 may affect bilayer properties (Fig. 5A). In our initial investigations, we performed spontaneous membrane binding simulations by placing ten SS-31 peptides in the aqueous phase in random orientations at distances of 1-3 nm from the bilayer surface and conducting simulations for up to $1.6 \mu \mathrm{s}$. Throughout each trajectory, we quantified the distance between each side chain and the bilayer center of mass along an axis perpendicular to the membrane (the Z-coordinate, Fig. S11 and Fig. 5B). We observed rapid association (<200 ns) of all peptides to the bilayer surface, in which all side chains had docked to the membrane near the interfacial region, presumably driven by the electrostatic attraction between the polybasic SS-31 and the negative surface charge of the lipid bilayer. However, the membrane association time differed among the side chains. For example, the $\mathrm{N}$ terminal Arg adsorbed to the bilayer rapidly (within $50 \mathrm{~ns}$ ), whereas the C-terminal Phe took significantly longer to localize to the bilayer (within $180 \mathrm{~ns}$ ) (Fig. 5B).

From these simulations, we observed two main types of SS-31 poses on the membrane (Fig. 5C): a peptide approach state in which the primary points of contact with the membrane are the $\mathrm{N}$-terminus and Arg side chain anchored to phosphate headgroups, and a peptide burial 
state in which the aromatic side chains have partitioned into the nonpolar core while the basic side chains remain electrostatically tethered to headgroup phosphates. Notably, the burial of the aromatic side chains of SS-31 in the acyl chain region is consistent with previous NMR studies (10). Once the peptides reached the burial state, each side chain assumed a distinct, stable membrane insertion depth, quantified as the average $z$-coordinate between the side chain and the membrane center of mass $\left(z^{\text {pos }}\right)$ (Fig. S11B,C). In the 20:80 TOCL:POPC system, the basic side chains occupied the interfacial region, with $\operatorname{Arg}\left(z^{\text {pos Arg }}=1.75 \mathrm{~nm}\right)$ residing just below the average position of lipid phosphates $\left(z^{\text {pos Phos }}=1.98 \mathrm{~nm}\right)$ and Lys residing at a slightly more distal position near the headgroups $\left(z^{\text {pos Lys }}=1.99 \mathrm{~nm}\right)$. By comparison, the aromatic residues assumed stable positions within the nonpolar core with the Phe side chain burying deeper $\left(z^{\text {pos }}\right.$ Phe $=1.12 \mathrm{~nm})$ than the $2^{\prime}, 6^{\prime}-\mathrm{Dmt}$ side chain $\left(z^{\text {pos DmT }}=1.37 \mathrm{~nm}\right)$. These residue-specific membrane insertion depths were consistent across the three lipid compositions tested.

To evaluate the association of SS-31 side chains with lipid headgroups, we analyzed radial distribution profiles from our MD trajectories (Fig. 5D, Fig. S12A). This analysis revealed that basic side chains of SS-31 preferentially interacted with anionic lipids. For example, based on $\mathrm{g}(\mathrm{r})$ peak height, in lipid systems with $20 \%$ TOCL, Arg and Lys showed 2.25- and 1.72-fold relative increases in local concentration of phosphate groups from TOCL compared with those from POPC (Fig. 5C). These results are consistent with analysis of peptide-lipid co-diffusion, suggesting that the SS-31 Arg can concurrently associate with multiple lipid phosphates with a preference for TOCL over POPC (Fig. S12B). As a complement to these analyses, we evaluated how the presence of SS-31 may influence lipid bilayer properties. First, lipid-to-lipid radial distribution profiles showed a slight peptide-dependent local clustering of anionic lipids (Fig. S12C). Second, based on the lateral mean square displacement of lipid phosphates, we observed marked peptide-dependent decreases in the lateral diffusivity $\left(D_{\mathrm{xy}}\right)$ of all tested lipids (Fig. S13). Finally, to study how SS-31 binding could affect membrane packing defects, we measured the solvent accessible surface area (SASA) of acyl chains in systems with and without SS-31 (Fig. S14). This analysis showed peptide-dependent decreases in acyl chain solvent accessibility of all tested lipids, consistent with our observed effects of SS-31 on interfacial hydration and packing density (Fig. 4C). Hence, taken together, our MD simulations elucidate the nature of polar and nonpolar interactions that mediate the SS-31-lipid bilayer interaction, and how peptide binding may affect lipid distribution, reduce lateral lipid mobility, and decrease the accessibility of solvent (and solvated ions) to acyl chains that may otherwise be exposed to the interface by packing defects. 


\section{SS peptide interactions with mitochondrial membranes}

Having evaluated the binding of SS-31 with model membranes, the next objective was to quantitatively assess how this peptide interacts with mitochondria. We first evaluated peptide binding interactions. The spectral complexity of mitochondria precludes analysis of SS-31 binding by endogenous peptide fluorescence, so we used instead the variant [ald]SS-31, which contains the environment-sensitive fluorophore aladan (44) in place of the C-terminal Phe residue (Fig. 1C). As shown previously, [ald]SS-31 displays a blue-shifted emission spectrum and an increase in fluorescence intensity when bound to bilayers, making it an excellent reporter for membrane interaction. This peptide variant has also been shown to target mitochondria in a manner similar to SS-31 $(11,12)$. We first thoroughly evaluated the binding of [ald]SS-31 to model membranes composed of POPC with TOCL, MLCL, and POPG in different molar ratios and under different ionic conditions (Fig. S15 A,B). Equilibrium binding parameters for [ald]SS-31 with model membranes compared favorably with those of SS-31 (Fig. S15C). Hence, this variant served as a suitable model for SS-31 binding to mitochondria.

In our evaluation of SS-31 mitochondrial interactions, our first goal was to assess the $\mathrm{CL}$-dependence of peptide binding. Because of the relative simplicity of the CL biosynthesis and remodeling pathway in yeast compared with higher eukaryotes (Fig. S1B), S. cerevisiae is an excellent model organism to directly compare effects of altered CL metabolism among otherwise isogenic systems. We therefore used mitochondria isolated from $S$. cerevisiae strains with normal CL metabolism, or with knockouts in cardiolipin synthase $(\Delta c r d 1)$ or the transacylase tafazzin ( $\Delta \operatorname{taz} 1)$ (45). Shotgun lipidomics data confirmed the expected phospholipidome of mitochondria from these three strains (Fig. 6A, left) - namely, that $\Delta c r d 1$ mitochondria lack $C L$ and have a buildup of $P G$, the immediate biosynthetic precursor of $C L$; and that $\Delta \operatorname{taz} 1$ mitochondria show an accumulation of MLCL and reduced CL. Further, the acyl chain distribution of anionic lipids among these strains shows the expected patterns of fatty acid saturation (45) (Fig. S16A). Emission scans of [ald]SS-31 revealed spectral changes with increasing mitochondria consistent with membrane interaction (Fig. S16B and Fig. 6A, right), showing two important features. First, as shown previously (8), collapse of the transmembrane potential $\left(\Delta \psi_{\mathrm{m}}\right)$ by the ionophore valinomycin had no effect on peptide interaction (Fig. 6A, inset). Second, the relative binding of [ald]SS-31 was nearly identical among the WT, $\Delta c r d 1$, and $\Delta \operatorname{taz} 1$ strains. Similar peptide binding between WT and $\Delta \operatorname{taz} 1$ strains is expected, given that tetra-acyl TOCL and tri-acyl MLCL equally support SS-31 binding in our reductionist systems (Figs. 2 and 3). Uninhibited peptide binding by the $\Delta c r d 1$ strain is perhaps surprising given that 
POPG supports significantly less peptide binding than CL (Figs. 2 and 3). However, our lipidomics data indicate that PG levels are dramatically increased when CL synthesis is blocked. This increase in PG likely creates a negative surface charge density of the MIM large enough to promote peptide binding at WT levels. Again, this supports a model in which SS-31 interaction is related to surface charge, not headgroup identity.

The second goal of these binding experiments was to directly compare peptide binding between model membranes and mitochondria. We reasoned that the best comparison would come from quantifying [ald]SS-31 binding as a function of a common independent variable (CL composition), made possible using the known mitochondrial CL concentration from our lipidomics data. When evaluated in this way, the binding curves of [ald]SS-31 to LUVs and WT mitochondria are strikingly similar (Fig. 6B). This comparison, although indirect, is consistent with a model in which binding of SS-31 to mitochondria is governed by the same interactions in our model systems; that is, through lipid bilayer interactions.

The final goal of these experiments was to evaluate the effect of SS peptides on the surface electrostatic properties of mitochondrial membranes. Based on our observation that SS peptides attenuated the $\psi_{s}$ of model membranes (Fig. 3C,D), we again used the ANS fluorescent reporter to measure the $\psi_{\mathrm{s}}$ of mitoplasts (mitochondria with osmotically ruptured outer membrane to allow probe access to the MIM). We therefore compared the titration of mitoplasts with SS-31 and mono- and divalent cations $\left(\mathrm{K}^{+}\right.$and $\mathrm{Ca}^{2+}$, respectively) to evaluate the relative effects of each species on ANS-detected $\psi_{\mathrm{s}}$ (Fig. 6C). All three cationic species caused an increase in ANS fluorescence, reflecting a decrease in the $\psi_{s}$ of mitochondrial membranes from WT (left) and $\Delta c r d 1$ (right) yeast. Consistent with the exponential dependence of formal charge on accumulation of ionized species in the electric field of membranes (Eq. $\mathrm{S} 11$ ), the effective concentration ranges of SS-31, $\mathrm{Ca}^{2+}$, and $\mathrm{K}^{+}$progressively differed by about two orders of magnitude (Fig. 6C, insets). The first notable feature of this analysis is the relative effect of each species on surface potential. Compared with $\mathrm{K}^{+}, \mathrm{Ca}^{2+}$ ions caused a much stronger impact on the relative $\psi_{\mathrm{s}}$, as expected. Interestingly, however, within its effective concentration range, SS-31 had a reduced effect on the relative $\psi_{\mathrm{s}}$ that was closer to that of the monovalent cation - an observation consistent with the fact that SS-31 does not completely reverse negative surface potential of model membranes even upon binding saturation (Fig. 3C). The second notable feature of this analysis is the effect of SS-31 on $\mathrm{Ca}^{2+}$-mediated decreases in the relative $\psi_{\mathrm{s}}$. SS-31 addition to WT mitoplasts prior to $\mathrm{CaCl}_{2}$ titration strongly blunted the effects of $\mathrm{Ca}^{2+}$ on surface potential (Fig. 6C, left, compare green and yellow symbols), 
supporting a binding model in which SS-31 preferentially binds to mitochondrial membranes over $\mathrm{Ca}^{2+}$ ions. Taken together, we conclude from the results of Fig. 6 that the binding of SS peptides to mitochondrial membranes is strongly dependent on surface charge of the lipid bilayer, that SS peptide binding causes a "controlled down-tuning" of the electric field originating from the membrane surface, and that SS peptides at the membrane interface can mitigate the effects of polyvalent cations on membrane surface electrostatics.

\section{The effects of SS peptides on cation accumulation at the membrane interface}

The final analyses of this study were focused on testing the functional implications of our observed effects of SS peptides on membrane surface electrostatics. We began with a theoretical consideration of how SS peptides may affect the decay of the electric field and ion distribution at the membrane surface based on GCS formalism (Eq. S10-S12) (Fig. 7A). By this analysis, SS peptides (modeled as trivalent ions at a bulk concentration in the $\mathrm{nM}$ range) accumulate at the membrane surface with the effect of: (i) reducing the magnitude of the surface charge, and (ii) decreasing accumulation of other cationic species at the interface. This effect is particularly relevant for $\mathrm{Ca}^{2+}$, because mitochondria serve as high-capacity calcium stores in mediating cellular ion homeostasis and $\mathrm{Ca}^{2+}$ overload can cause severe damage to anionic lipid bilayers, particularly within mitochondria (see Discussion). The analysis of Fig. 7A shows that SS peptides cause a strong reduction in surface accumulation of divalent cations, by over an order of magnitude. Hence, this model, albeit a simplified one, makes testable predictions about how SS peptides may mitigate divalent cation stress by reducing $\mathrm{Ca}^{2+}$ accumulation at the membrane interface. Our model was tested as follows.

First, we measured the effects of SS peptides on the accumulation of $\mathrm{Ca}^{2+}$ in the interfacial region of model membranes (Fig. 7B-D). To this end, we used a fluorescence-based approach with Calcium Green-5N (CG-5N), a membrane-impermeant probe that exhibits an increase in emission intensity upon binding $\mathrm{Ca}^{2+}$ (characterized for our system in SI Results $\mathbf{H}$ and Fig. S17A,B). We reasoned that the binding of $\mathrm{Ca}^{2+}$ to anionic lipid bilayers in the headgroup region would reduce the bulk $\mathrm{Ca}^{2+}$ detected by the $\mathrm{CG}-5 \mathrm{~N}$ probe, providing a direct readout of $\mathrm{Ca}^{2+}$ accumulation at the membrane interface. As a proof of principle, we titrated $\mathrm{Ca}^{2+}$ into solutions of CG-5N containing LUVs with monoanionic (POPG) or dianionic (TOCL) lipids at different concentrations (Fig. 7B). In the absence of LUVs, there was a saturating increase in CG-5N emission as the probe became maximally bound with $\mathrm{Ca}^{2+}$. However, the presence of POPG- and TOCL-containing LUVs caused a reduction in the CG-5N response to $\mathrm{Ca}^{2+}$ that was directly related to the amount of bilayer surface charge in the system. Hence, this 
assay provided a direct measure of $\mathrm{Ca}^{2+}$ sequestration within the headgroup region of anionic bilayers. To test the effects of SS peptides on $\mathrm{Ca}^{2+}$ binding to membrane surfaces, we first titrated SS-20 and SS-31 into solutions containing LUVs with 20\% TOCL pre-bound with $\mathrm{Ca}^{2+}$ (Fig. 7C). These results showed that SS-20 and SS-31 released $\mathrm{Ca}^{2+}$ from the membrane interface in a dose-dependent manner that was identical between the two peptides. Importantly, the CG-5N response began to saturate near $P: L^{\text {eff }}$ of $\sim 1: 10$ (Fig. 7C, green traces), corresponding to the peptide binding saturation point, therefore supporting that $\mathrm{Ca}^{2+}$ release into the bulk corresponded to peptide bilayer binding. As a complementary approach, we performed $\mathrm{Ca}^{2+}$ titration on solutions of LUVs that had been pre-incubated without peptide or with SS peptides at molar $P: L^{\text {eff }}$ of 1:20 and 1:10 (Fig. 7D). These results confirmed that the presence of SS-20 and SS-31 both reduced the equilibrium binding of $\mathrm{Ca}^{2+}$ to anionic bilayer surfaces in a dose-dependent manner. Taken together, these results support a model in which SS peptide binding to bilayer surfaces reduces the accumulation of $\mathrm{Ca}^{2+}$ ions within the interfacial region of model membranes.

Second, we measured the effects of SS peptides on mitochondrial $\mathrm{Ca}^{2+}$ flux (Fig. 7E-G). External $\mathrm{Ca}^{2+}$ is taken up into the mitochondrial matrix electrophoretically by the $\Delta \psi_{\mathrm{m}}$ (matrix negative). We reasoned that if SS peptides altered the accumulation of cations at the bilayer surface (Fig. 7B-D), then they may have a measureable effect on mitochondrial $\mathrm{Ca}^{2+}$ uptake. We therefore performed time course measurements of isolated mitochondria supplemented with respiratory substrate $\mathrm{NADH}$ followed by additions of calcium. In these experiments, we monitored the relative $\Delta \psi_{\mathrm{m}}$ using the potentiometric probe TMRM (the emission of which is inversely related to membrane potential, Fig. S17C) and in parallel experiments we monitored the external $\left[\mathrm{Ca}^{2+}\right]$ using CG-5N (Fig. 7E). Note that because S. cerevisiae mitochondria do not contain a $\mathrm{Ca}^{2+}$ uniporter, $\mathrm{Ca}^{2+}$ uptake does not occur spontaneously but can occur in the presence of a calcium ionophore, for which we used ETH-129. These time courses show two key processes: (i) following NADH addition, there is a TMRM-detected establishment of the $\Delta \psi_{\mathrm{m}}$, and (ii) after each calcium addition, there is a CG-5N-detected spike in external $\left[\mathrm{Ca}^{2+}\right]$ followed by a decrease in CG-5N emission as $\mathrm{Ca}^{2+}$ is taken into the matrix, which is accompanied by a transient TMRM-detected partial depolarization and re-establishment of the $\Delta \psi_{\mathrm{m}}$ (see Fig. S18A for expanded views of each of these transient processes). The absence of dynamic changes in TMRM and CG-5N emission in samples lacking ETH-129 (Fig. 7E, "no ETH-129") confirmed that these spectral changes did indeed reflect $\mathrm{Ca}^{2+}$ uptake. These experiments revealed two notable effects of SS peptides. The first was related to the establishment of the $\Delta \psi_{\mathrm{m}}$ in samples containing $\mathrm{ETH}-129$. Upon reaching a threshold potential, 
there was a temporal lag in potential generation, after which the mitochondria resumed establishing the maximal $\Delta \psi_{\mathrm{m}}$. This is attributable to the energetic demands coupled to the uptake of calcium that exists in the external buffer of these mitochondrial samples (see also Fig. S17D). Interestingly, both SS-20 and SS-31 reduced the extent of this temporal delay in mitochondrial energization observed in the presence of increasing $\left[\mathrm{Ca}^{2+}\right.$ (Fig. $\mathbf{7 F}$ and Fig. S18A, " $\Delta \psi_{\mathrm{m}}$ establishment"). Quantitative analysis of $\mathrm{Ca}^{2+}$-dependent $\Delta \psi_{\mathrm{m}}$ generation (described in Fig. S18B) revealed that SS-31 reduced this temporal delay in a dose-dependent and saturable manner (Fig. 7G). The second related effect was associated with subsequent calcium spikes. Following calcium additions, mitochondria transiently depolarized to near the same $\Delta \psi_{\mathrm{m}}$ associated with the temporal lag. The kinetics of repolarization during the first calcium additions were faster in the presence of SS peptides; however, with increasing calcium load, the peptides no longer enhanced repolarization (see Fig. S18A, "Ca ${ }^{2+}$ transient 1-4"). By contrast, external calcium flux, judging by the extent of the CG-5N signal and by the kinetics of $\mathrm{Ca}^{2+}$ uptake, was not affected at all by SS peptides. Hence, under these conditions, SS peptides do not measurably alter the calcium storage capacity of mitochondria, but do improve the ability to respond energetically to calcium load. These results may provide a mechanistic underpinning to previous observations that acute treatment of isolated mitochondria with SS peptides protected against $\mathrm{Ca}^{2+}$-induced onset of the mitochondrial permeability transition (MPT) (8).

Finally, we measured the effects of SS peptides on mitochondrial calcium stress that may occur independently of $\mathrm{Ca}^{2+}$ uptake into the matrix (Fig. $7 \mathbf{H}-\mathbf{J}$ ). To this end, we used a ${ }^{31} \mathrm{P}$ ssNMR-based strategy to monitor mitochondrial membrane structural integrity, based on previous studies using NMR analysis of membranes from prokaryotic cells (46) or isolated mitochondria (47-49). We first characterized ${ }^{31} \mathrm{P}$ ssNMR spectra of our isolated mitochondria in comparison to model membranes (SI Results $\mathbf{H}$ and Fig. S18), showing that mitochondria spectra contained a complex superposition of lineshapes that could be deconvoluted for the direct analysis of phospholipid bilayers. Based on previous work using NMR analysis to measure the effects of calcium (up to $10 \mathrm{mM}$ ) on mitochondrial membranes (49), we then tested the ability of SS-31 to mitigate damage to mitochondrial membranes by calcium stress. We found that acute treatment of mitochondria with SS-31 $(8.5 \mathrm{nmol}$ peptide per $\mathrm{mg}$ mitochondrial protein) reduced NMR-detected membrane degradation caused by high calcium load (up to 5 $\mathrm{mM} \mathrm{CaCl}_{2}$ ) (Fig. 7H). By spectral deconvolution, we observed that SS-31 treatment resulted in a significant preservation of the L2 peak, which corresponds to lamellar phospholipid bilayers (Fig. 7l, Fig. S19). To corroborate these findings, we measured mitochondrial oxygen consumption rates under similar conditions of SS peptide treatment and calcium stress. Under 
these conditions, we observed a significant increase in basal respiration caused by calcium addition that was prevented by SS-31 pretreatment (Fig. 7J). Hence, acute SS-31 treatment rendered protective effects under these harsh conditions of calcium stress manifested as the preservation of membrane integrity and OXPHOS activity.

\section{Discussion}

It is well established that SS-31 specifically targets mitochondria and ameliorates the decrease in mitochondrial function associated with aging, cellular stress, and heritable diseases. Further, SS-31 is known to have affinity for aqueous dispersions of anionic lipids (12), particularly membranes containing CL (10). However, the nature of the interaction between SS31 and mitochondrial membranes has remained elusive. Toward the goal of understanding the molecular MOA of SS-31, the present study addressed how this peptide interacts with lipid bilayers and the effect that it has on membrane properties. Our results support a mechanism in which the modulation of mitochondrial membrane surface electrostatic properties plays a critical role.

The first key finding of this study was on the lipid determinants of SS-31 membrane interactions, based on empirical binding isotherms with model membranes (Figs. 2, S15) and MD simulations (Figs. 5, S11). Consistent with previous results $(10,12)$, we found that SS-31 had negligible interaction with membranes composed solely of zwitterionic lipids, and required anionic lipids for saturable binding. Independent lines of evidence in this study support that the primary determinant of SS-31 binding is the number of negative charges within the lipid headgroup region. First, the binding of SS-31 to membranes containing tetra-acyl CL (TOCL) or its lysolipid variant (MLCL) was virtually indistinguishable, as demonstrated in model membranes (Fig. 2) and isolated mitochondria (Fig. 6A, WT vs. $\Delta$ taz1). Notably, this supports the ability of SS-31 to act as a therapeutic for the treatment of Barth syndrome, which is characterized by an increase in the MLCL/CL ratio of the MIM (24). Second, membranes containing monoanionic PG supported roughly half of the SS-31 binding capacity observed in membranes with equal concentrations of CL (Fig. 2). In this regard, mitochondria lacking CL biosynthesis sustained near-WT levels of peptide binding because PG was increased to roughly twice the normal CL concentration (Fig. 6A, WT vs. $\Delta c r d 1$ ). Together, these results support a model in which SS peptide binding to lipid bilayers depends on the anionic surface charge density, not the identity of a particular component lipid. This model is supported by our atomistic MD work, showing that bilayer-docked SS-31 resides stably in the interfacial region with similar residue-specific penetration depths and lipid interactions among TOCL, MLCL, and POPG- 
containing bilayers (Figs. 5, S11, S12). Yet based on ITC measurements, the noncovalent interactions that mediate SS-31 binding appear to be dominated by entropy rather than by the energy of polar contacts (Fig. 2D,E), underscoring the importance of nonpolar interactions between aromatic side chains of the peptide and the acyl chain region in determining binding stability. Therefore, a key role of the bilayer surface charge is likely the establishment of a strong electric field (see Fig. S2) that increases the effective concentration of peptides in the interfacial region, where they are then able to establish binding interactions, which are dominated by hydrophobic contacts (Fig. 8A). We note that although $C L$ is not strictly required for SS-31 binding, it is likely essential for peptide action within mitochondrial membranes for two main reasons. First, under conditions of normal lipid metabolism, CL is the lipid that contributes the most to the strong negative surface charge of the MIM. As shown by our lipidomics data (Fig. 6A), the concentration of dianionic $C L$ is greater than that of other monoanionic phospholipids (PS, PA, PG); other highly charged lipids including phosphoinositides (the phosphorylated variants of $\mathrm{PI}$ ) are generally confined to cytoplasmic leaflets of the endomembrane system (50) and not abundant in mitochondria. Second, the inverted conical molecular geometry of $\mathrm{CL}$, promoted by small headgroup volume and highly unsaturated fatty acids (cf. Fig. S16A), creates packing defects in CL-rich bilayers, which may be critical for providing peptide side chain access to acyl chains. As a corollary, specific physicochemical features of CL may be essential for downstream (post-binding) effects of SS peptides on membrane properties (see below). Hence, considering that polypeptide binding to lipid bilayers is determined by a combination of surface charge density and packing defects (51), we propose that SS peptides target the specialized environment of the MIM through a combination of a strong surface electrostatics and lipid packing defects imparted by features such as high [CL], highly unsaturated acyl chains, and low sterol content.

The second key finding of this study was the equilibrium membrane binding parameters of SS-31 and how they elucidate the molecular MOA. First, the lipid:peptide stoichiometry $(n)$ serves as an index for peptide binding density ( $n$ scales inversely with the number of peptides bound per unit membrane area). We show that under conditions of maximal peptide binding density, model membranes composed of 20 mol\% CL and 20 mol\% PG bind SS-31 with $n \approx 6-7$ (area occupancy $\sim 500-600 \AA^{2}$ ) and $n \approx 15$ (area occupancy $\sim 1000 \AA^{2}$ ), respectively. To put these values into context, consider a typical cell with a total mitochondrial volume of $2 \times 10^{3} \mu \mathrm{m}^{3}$ and a total MIM surface area of $2 \times 10^{5} \mu \mathrm{m}^{2}$ (52). Assuming, conservatively, that $10 \%$ of the MIM surface is available for peptide binding, this translates into a mitochondrial SS-31 concentration of $400 \mu \mathrm{M}$ at maximal binding density. This estimate, although approximate, is consistent with 
the observed 1000 to 5000 -fold mitochondrial accumulation in cells incubated with low nM amounts of peptide (8). Second, the binding affinity $\left(K_{D}\right)$ of SS-31 for membranes was found to be in the low $\mu \mathrm{M}$ range. Compared with typical drug compounds that bind molecular targets with much higher affinity (e.g., $K_{\mathrm{D}}$ in the $\mathrm{nM}$ and lower range), the binding affinity of SS peptides to membranes is very weak. This low affinity range is to be expected, considering that the primary mode of peptide interaction is with the thermally disordered lipid bilayer rather than with a molecular binding pocket with a defined covalent structure. Furthermore, the low $\mu \mathrm{M}$ affinity of SS-31 for membranes is consistent the pharmacokinetic profile of SS peptides, showing rapid clearance of the peptide after administration (13) as well as peptide release from cultured cells upon media exchange (7). Notably, the correspondence between SS-31 binding parameters with model membranes and with isolated mitochondria observed in this study supports a model in which the primary mode of SS peptide interaction is with lipid bilayers. Notably, it remains possible (and perhaps likely) that a fraction of SS peptides may interact with proteins (e.g., within acidic pockets) and/or at protein-lipid interfaces, particularly given the protein-rich nature of mitochondrial membranes.

The third key finding of this study addressed the effects of SS peptides on membrane physical properties. Using ${ }^{31} \mathrm{P}$ NMR and SAXS, we found that SS-31 did not disturb the lamellar bilayers of model LUVs, even at high peptide binding densities (Fig. 4A,B). This is consistent with previous work (8) and the present study (Fig. S17E), showing that SS peptides, even at high concentrations, do not dissipate ion gradients $\left(\Delta \Psi_{m}\right)$ of respiring mitochondria. As noted above, given their amphipathic character, SS peptides resemble AMPs. This shared amphipathicity likely drives the interactions with bacterial membranes (AMPs) and membranes of bacterial origin (SS peptides). We propose that the short (four-residue) length of SS peptides is the predominant feature that keeps them from disrupting membrane structure, unlike AMPs, which are significantly longer (40). SS-31 did, however, affect properties of the interfacial membrane region in a dose-dependent manner. First, based on membrane-bound solvatochromic probes, SS-31 caused a modest but repeatable increase in lipid packing, particularly for CL-containing bilayers (Fig. 4C), a result consistent with our analysis of peptidedependent reduction in acyl chain solvent accessible surface area (Fig. S14). Due to its conical molecular geometry, CL can cause localized exposure of acyl chains to the aqueous interface (27). Intercalation of SS-31 aromatic chains into these pockets could fill such interfacial "voids" (packing defects), which could in turn have implications for bilayer properties such as mechanostability and elasticity. Second, based on computational approaches, we found that SS-31 caused a reduction in the lateral diffusivity of lipids (Fig. S14), consistent with a model in 
which peptide binds tightly enough to anionic lipids that they co-diffuse for significant (at least $\mu \mathrm{sec})$ time scales. Third, using electrokinetic and fluorescence-based approaches, we found that SS-31 decreases the surface charge (and hence the $\psi_{\mathrm{s}}$ ) of both model (Fig. 3C,D) and mitochondrial (Fig. 6C) membranes. All of these observed effects are likely interrelated. For example, by partially neutralizing the charge of anionic lipids, the binding of SS-31 will reduce the hydration and effective cross-sectional area of headgroups, as well as decrease inter-lipid electrostatic repulsion, all of which could increase lipid packing. We therefore propose a model for the molecular MOA of SS-31 in which the primary effect of bilayer interaction is the downregulation of the $\psi_{\mathrm{s}}$ of mitochondrial membranes. In support of this model, we found that SS peptides caused the partial neutralization of the strong negative surface charge density of mitochondrial membranes, but appeared to do so in a way that does not cause positive charge overcompensation (Figs. $3 \mathrm{C}$ and 6C). A critical corollary of this model is that this effect on surface electrostatics should be shared by all SS peptides due to their common positive charge density, and not depend on specific side chain features such as the ROS scavenging potential of the 2',6'-Dmt moiety of SS-31. Indeed, we found that SS-20 and SS-31 are indistinguishable in terms of their effects on tuning membrane surface potential.

In this model, it is important to consider how peptides may distribute within the membrane interface. SS peptide occupancy at or near the surface of anionic membranes is driven by the high $(+3)$ positive charge density that dictates peptide equilibrium distribution in the electrostatic field of the bilayer and promotes ionic interactions with negatively charged membrane-bound groups. Hence, SS peptides bind the membrane surface with micromolar affinity that is entropically and enthalpically favored (Fig. 2); however, given that the binding is relatively weak and reversible depending on local ionic strength (Fig. 3), a fraction of these small polybasic amphiphiles are likely to exist untethered to the membrane surface, but stably reside within the electric field of the double diffuse layer of the anionic mitochondrial membranes (Fig. 8B). This distribution is likely to vary laterally along the MIM in different mitochondrial subcompartments that have different surface charge characteristics. Noting that ion distribution near a charged membrane surface is an exponential function of formal charge (Eq. S11), the polybasic nature of SS peptides is critical in allowing them to bind anionic bilayers in competition with physiological concentrations of mono- and divalent cations (trivalent cations are rare in biological systems). Hence, in accordance with GCS theory (Fig. 7A), the accumulation of SS peptides at the interface will have the effect of shifting the balance of other ionic species (e.g., solvated metal ions and amphitropic basic proteins) away form the membrane surface. Finally, given that CL may be enriched on the inner leaflet of the MIM (53), SS peptides may 
preferentially accumulate on the matrix-facing side of this membrane, provided that a pathway exists for them to traverse the MIM. Ongoing work in our group is aimed toward understanding the dynamic distribution of SS peptides among mitochondrial subcompartments.

How might the down-regulation of the $\psi_{\mathrm{s}}$ of anionic mitochondrial membranes underpin the broad therapeutic efficacy of SS peptides? We propose several nonexclusive possibilities (Fig. 8C). First, reducing the magnitude of the membrane surface charge will alter cation distribution at the interfacial region. This is particularly relevant for $\mathrm{Ca}^{2+}$ because mitochondria must accumulate large concentrations of this divalent cation in their role as cellular calcium stores. $\mathrm{Ca}^{2+}$ binding to the lipid headgroup region severely alters properties of anionic bilayers (48). More relevant to mitochondrial membranes, $\mathrm{Ca}^{2+}$ interacts strongly with $\mathrm{CL}$, altering bilayer properties (e.g., demixing, phase behavior, and headgroup conformation) (19); $\mathrm{Ca}^{2+}$ can disrupt protein-lipid interactions, causing effects such as respiratory complex disintegration (54); and mitochondrial $\mathrm{Ca}^{2+}$ dyshomeostasis underpins a wide variety of mitochondrial diseases (55). In this study, we have directly shown that the binding of SS peptides reduces the effects of $\mathrm{Ca}^{2+}$ on mitochondrial surface electrostatics (Fig. 6C) and reduces the equilibrium binding of $\mathrm{Ca}^{2+}$ to the interface of anionic synthetic membranes, which act as a "sink" for divalent cations (Fig. 7B-D). We also demonstrated that SS peptides appear to reduce the energetic burden associated with calcium uptake (Fig. 7E-F) and preserve mitochondrial function and membrane integrity with calcium stress (Fig. 7G-I). Although we do not directly show the latter two physiological effects originate specifically from the reduction of $\mathrm{Ca}^{2+}$ accumulation at the bilayer surface, evidence that the site of action of SS peptides is at the membrane interface supports this as a likely mechanism. This proposed effect of SS peptides on mitigating bilayer $\mathrm{Ca}^{2+}$ accumulation is consistent with their demonstrated efficacy toward processes coupled to pathogenic mitochondrial $\mathrm{Ca}^{2+}$ overload, including ischemia-reperfusion, induction of the MPT, and apoptosis/necrosis (5). A second related mechanism is that reducing the magnitude of the $\psi_{\mathrm{s}}$ could reduce the mitotoxic interaction of basic proteins with CL-rich mitochondrial membranes. These include cytochrome $c$ (cyt. $c$ ), whose binding to the MIM leads to lipid peroxidation and initiates the intrinsic apoptotic pathway (56), and neurotoxic amyloid peptides, known to aggregate upon and damage mitochondrial membranes $(57,58)$. To this point it is noteworthy that SS-31 has been shown to alter cyt. $c$ interactions with CL-containing bilayers $(10,11)$ and to reduce mitochondrial damage in models of Alzheimer's and Parkinson's disease (59, 60). Third, by downregulating the surface charge density of mitochondrial membranes, SS peptides may act in part by altering the physical properties of CL-containing bilayers. Full ionization of CL phosphate groups creates strong electrostatic repulsion in the lipid headgroup region and 
increases the effective headgroup area, imparting a more cylindrical lipid geometry. Reduction of $\mathrm{CL}$ headgroup charge promotes more conical molecular geometry, thereby altering lipid packing interactions and making the establishment of membrane curvature more energetically accessible (20,61-63). SS peptides may therefore help stabilize local regions of high curvature in the morphologically complex MIM. A final potential mechanism could relate to recent findings that dysfunctional mitochondrial protein complex assembly is coupled to the release of CL into the bulk lipid phase (64); under such pathological conditions, SS peptides could help regulate the resulting increase in membrane surface charge.

With these proposed mechanisms in mind, there are two ways to consider the broad efficacy of SS peptides in treating mitochondrial dysfunction. First, the controlled downregulation of the mitochondrial $\psi_{\mathrm{s}}$ could have direct and specific impacts on different molecular pathomechanisms: for instance, by altering the molecular geometry of MLCL to impart more conical-like character, SS peptides could help stabilize cristae curvature in patients with Barth syndrome. But from a more general standpoint, SS peptides could also serve to reduce the constant stresses that mitochondria face under normal physiological conditions, including spikes in calcium levels and mitotoxic interactions between CL and polybasic proteins. Such stresses could present a manageable burden on mitochondria of healthy individuals, but an excessively taxing one on mitochondria whose functional integrity is compromised, as occurs with ageing and heritable mitochondrial disease. Hence, the remarkably broad effects of SS peptides in treating mitochondrial dysfunction could also be based partly on their ability to mitigate general molecular stressors at the membrane.

In summary, the results of this study support a model in which SS-31 binding to mitochondrial membranes is largely driven by, and subsequently downregulates, the $\psi_{\mathrm{s}}$ as a core component of its molecular MOA. Considering SS peptides as mitochondria-targeted hydrophobic polyvalent cations, this model explains how these compounds act upon the general property of bilayer surface electrostatics to serve as broad-based therapeutic compounds for a wide array of mitochondrial disorders. 


\section{Acknowledgements}

The authors would like to thank Dr. Steven Claypool (Johns Hopkins University School of Medicine) for the original synthesis of yeast mutants used in this study, Drs. Mu-Ping Nieh and Kuo-Chih Shih (University of Connecticut) for technical assistance with SAXS measurements, Dr. Georg Pabst (University of Graz) for providing the GAP program for analysis of SAXS data, and the UT Health-San Antonio Mass Spectrometry Core Facility. This work was supported by the National Institutes of Health (R01-GM113092 to N.N.A., R35-GM1197623 to E.R.M., and RF1-AG061872 to X.H.), by the National Science Foundation (GRFP award 1247393 to K.B.) and by a charitable contribution from the Social Profit Network (to N.N.A.)

\section{Figure Legends}

Fig. 1. SS peptide chemical structures. (A) SS-31 (D-Arg-2'6'-dimethylTyr-Lys-Phe- $\mathrm{NH}_{2}$ ). (B) SS-20 (Phe-D-Arg-Phe-Lys-NH ${ }_{2}$ ). (C) [ald]SS-31, SS-31 with an aladan moiety (red) in place of Phe.

Fig. 2. SS-31 binding isotherms. $(A-C)$ Fluorescence-based measurements. $(A)$ Peptide titrations. Liposomes containing anionic lipid (20 mol\% TOCL, MLCL, or POPG) or POPC only at the lipid concentrations indicated were titrated with SS-31 (4 nmol increments). Values shown are means $(n=3 \pm S D)$ and lines show data fits based on Eq. S2. (B) Lipid titrations. Peptides at the concentrations indicated were titrated with liposomes (30 nmol lipid increments). Values shown are means $(n=3 \pm S D)$ and lines show data fits based on Eq. S3. (C) Scatchard analyses. Titration data with liposomes containing $20 \mathrm{~mol} \% \mathrm{TOCL}, \mathrm{MLCL}$, and POPG shown as Scatchard plots. Upper panel, peptide titrations (125 $\mu \mathrm{M}$ lipid) fit according to Eq. S6; lower panel, lipid titrations (10 $\mu \mathrm{M}$ SS-31, TOCL and MLCL; $5 \mu \mathrm{M} \mathrm{SS}-31$, POPG) fit according to Eq. S8. (D) ITC analyses. Wiseman plot showing blank-subtracted average integrated heats $(\mathrm{n}=3 \pm$ SD) as a function of lipid:SS-31 molar ratio for LUVs containing TOCL, MLCL, and POPG. $(E)$ Summary of binding parameter data. Statistical differences within each dataset determined relative to TOCL-containing membranes: $P<0.05 ; \bullet P<0.01$.

Fig. 3. SS-31 binding and surface electrostatics. $(A)$ Binding isotherms. Peptide was titrated with LUVs of the indicated composition (20\% TOCL, [SS-31] $=7.5 \mu \mathrm{M}$ and $20 \%$ POPG, [SS-31] $=3.8 \mu \mathrm{M}$ ) at $30 \mathrm{nmol}$ lipid increments in the presence of different concentrations of added $\mathrm{KCl}$. Values shown are means $(n=3 \pm S D)$ and lines show data fits based on Eq. S3. Because isotherms at higher $[\mathrm{KCl}]$ did not reach saturation, curves within each membrane set were fit globally assuming a common saturation point. $(B)$ Equilibrium binding parameters $n$ and $K_{\mathrm{D}}$ at different ionic strengths as a function of [KCl] (upper panels) and as a function of $\psi_{\mathrm{s}}$, calculated from GCS formalism, Eq. S10-S12 (lower panels) based on binding isotherms of panel $A$. (C,D) Effect of SS-31 binding on surface charge. Measurements of $(C)$ zeta potential $(\zeta)$ and $(D)$ ANS fluorescence shown as a function of [SS-31]:[Lip] ${ }^{\text {eff }}$ for membranes containing $20 \%$ TOCL and $20 \%$ POPG as indicated (values are means, $n=3 \pm S D$ ).

Fig. 4. Effects of SS-31 on structural properties of model membranes. $(A, B)$ SS-31 does not cause major structural perturbation of model membranes. LUVs composed of $20 \%$ anionic lipid (TOCL, MLCL, or POPG) in POPC background were measured in the absence or presence of peptide ([P]:[L] $\left.]^{\text {eff }}=1: 5\right)$ as indicated. $(A)$ Synchrotron SAXS measurements showing 
background-subtracted scattering density profiles for LUVs in the absence (black) or presence (red) of SS-31. (B) ${ }^{31} \mathrm{P}$ solid state NMR spectra of LUVs in the absence (lower spectra) or presence (upper spectra) of SS-31. (C) SS-31 alters lipid packing interactions. Changes in laurdan/prodan GP values $(\Delta G P)(n=3 \pm S D)$ with increasing [SS-31] for LUVs containing $20 \%$ TOCL or POPG in POPC background as indicated. Data and fits in grey show SS-31 fractional saturation (calculated from Fig. $2 \mathrm{~A}, 25 \mu \mathrm{M}$ lipid).

Fig. 5. Molecular dynamics simulations of SS-31 bilayer interactions. (A) Snapshot of a typical MD simulation with SS-31 peptides in the aqueous phase relative to the upper leaflet of a $20 \%$ TOCL bilayer. Peptides are shown in licorice representation (cyan, carbon; blue, nitrogen; red, oxygen), lipid acyl chains are in wireframe, and lipid phosphates are shown as van der Waals spheres for POPC (gray) and TOCL (red). (B) Temporal evolution of SS-31 Arg (blue) and Phe (green) side chain positions relative to the membrane center of mass (C.O.M.). Black lines show the average position of all phosphates in the upper leaflet, colored dots show $z$ coordinates $\left(z^{P O S}\right)$ of individual side chains, and colored lines represent average $z^{P O S}$ values. $(C)$ Orientation of SS-31 bound to a lipid bilayer consisting of $20 \mathrm{~mol} \%$ TOCL in a POPC background in the peptide approach (left) and buried (right) states. Peptide representation as in $(A)$, with all lipid phosphates shown as gray van der Waals spheres. $(D)$ Lipid distributions around basic SS-31 residues. Lateral ( $x-y$ plane) radial distribution profiles from Arg and Lys side chains to lipid headgroup phosphate atoms of TOCL or POPC as indicated.

Fig. 6. Interaction of SS-31 with isolated mitochondria. $(A)$ [ald]SS-31 binding with differing phospholipid composition. Left, Percentages of total phospholipid of each species (PC, phosphatidylcholine; PE, phosphatidylethanolamine; CL, cardiolipin; MLCL, monolyso-CL; PG, phosphatidylglycerol; PA, phosphatidic acid; PI, phosphatidylinositol; PS, phosphatidylserine; LPC, lyso-phosphatidylcholine) in mitochondria from WT, $\Delta t a z 1$, and $\Delta c r d 1$ strains measured by shotgun lipidomics. Right, binding of $1 \mu \mathrm{M}$ [ald]SS-31 with mitochondria isolated from WT, $\Delta t a z 1$, and $\Delta c r d 1$ yeast strains quantified as the mean fractional increase $\left(F / F_{0}\right)$ in aladan emission ( $n=3 \pm S D$ ) with increasing mitochondria concentration for fully energized mitochondria. Inset, relative binding of [ald]SS-31 to mitochondria $\left(0.2 \mathrm{mg} \mathrm{ml}^{-1}\right)$ in the presence or absence of $1 \mu \mathrm{M}$ valinomycin ("val") as indicated. (B) Comparison of [ald]SS-31 binding to mitochondria and model membranes. [ald]SS-31 binding is shown as a function of $C L$ concentration for: (i) LUVs of defined TOCL composition under high salt conditions (gray, Fig. S15) and (ii) mitochondria isolated from yeast WT strain (red, Figs. S16 and 6A). Calculation of [CL] for mitochondrial samples was based on lipidomics analyses of Fig. 6A. (C) Effects of SS31 and salt cations on mitochondrial surface potential. Fractional change in ANS emission is shown for titration mitoplasts from WT (left) and $\Delta c r d 1$ (right) yeast with SS-31, $\mathrm{CaCl}_{2}$, or $\mathrm{KCl}$ at the indicated concentrations $\left(n=3 \pm S D\right.$ ). For WT samples, $\mathrm{CaCl}_{2}$ titrations were also conducted following the addition of $20 \mu \mathrm{M}$ SS-31 (" $\mathrm{CaCl}_{2}+\mathrm{SS}-31$ "). Insets, ANS emission data plotted as a function of log concentration for all three titrants.

Fig. 7. SS peptide effects on $\mathrm{Ca}^{2+}$-membrane interactions and on mitochondrial cation stress. $(A)$ Profiles of surface potential decay (upper panel) and ion distribution (lower panel) based on GCS theory in the presence or absence of SS-31 assuming a lipid bilayer of $20 \%$ CL and bulk concentrations as follows: monovalent 1:1 electrolyte (e.g., $\mathrm{Na}^{+} \mathrm{Cl}^{-}$), $80 \mathrm{mM}$; divalent 2:1 electrolyte (e.g., $\mathrm{Ca}^{2+} \mathrm{Cl}^{-}$), $1 \mu \mathrm{M}$; and SS-31 (where present), $100 \mathrm{nM}$. (B-D) Effect of SS peptides on $\mathrm{Ca}^{2+}$ accumulation at model membrane interfaces. Time course measurements of CG-5N emission were performed as follows. $(B)$ The effect of anionic bilayers on the CG-5N response to added $\mathrm{Ca}^{2+}$ was evaluated by preparing solutions in the absence or presence of LUVs (20 mol\% TOCL or $20 \mathrm{~mol} \%$ POPG in a host POPC background) at the concentrations 
indicated. Starting at $\mathrm{t}=60 \mathrm{~s}, \mathrm{CaCl}_{2}$ was titrated in increments of $1 \mathrm{nmol}$ at $20 \mathrm{~s}$ intervals. $(C, D)$ The effect of SS peptides on interfacial $\mathrm{Ca}^{2+}$ binding was evaluated by preparing solutions with LUVs $\left(20 \mathrm{~mol} \%\right.$ TOCL, [Lip] $\left.{ }^{\text {eff }}=100 \mu \mathrm{M}\right)$ and $(C)$ subjecting samples to $5 \mathrm{nmol} \mathrm{CaCl}$ addition $_{2}$ (arrowhead, $t=60 \mathrm{~s}$ ) followed by addition of SS-31 or SS-20 at the indicated amounts at $20 \mathrm{~s}$ intervals, or $(D)$ pre-binding SS peptide at the indicated concentrations followed by titration of $\mathrm{CaCl}_{2}$ in increments of $1 \mathrm{nmol}$ at $20 \mathrm{~s}$ intervals starting at $\mathrm{t}=60 \mathrm{~s}$. (E-G) Effect of SS peptides on mitochondrial $\mathrm{Ca}^{2+}$ uptake. Time courses of mitochondria $\left(50 \mu \mathrm{g} \mathrm{ml}^{-1}\right)$ were performed in buffer containing TMRM $\left(\Delta \psi_{\mathrm{m}}\right.$ measurements) or CG-5N (external $\left[\mathrm{Ca}^{2+}\right]$ measurements). To facilitate comparison, both TMRM and CG-5N emission data were normalized relative to the full range of the probe response over the course of each measurement. (E) TMRM and CG-5N traces of $\Delta \psi_{\mathrm{m}}$ generation following addition of $1 \mathrm{mM} \mathrm{NADH}$ (black arrowheads) and of calcium transients following addition of $25 \mu \mathrm{M} \mathrm{CaCl}_{2}$ (open arrowheads) in samples pre-treated with or without 5 $\mu \mathrm{M}$ ETH-129, $25 \mu \mathrm{M}$ SS-20, and/or $25 \mu \mathrm{M}$ SS-31 as indicated. $(F)$ TMRM traces of $\Delta \psi_{\mathrm{m}}$ generation following addition of $1 \mathrm{mM}$ NADH (black arrowheads) in samples containing the indicated $\left[\mathrm{CaCl}_{2}\right]$ and supplemented with vehicle only (control), $25 \mu \mathrm{M}$ EDTA, SS-20, or SS-31 as indicated. (G) Dose response of SS-31 on $\Delta \psi_{\mathrm{m}}$ generation with $25 \mu \mathrm{M} \mathrm{CaCl}_{2}$ (defined in Fig. S18B, $\mathrm{n}=3 \pm \mathrm{SD})$. $(H-J)$ Effect of SS-31 on external $\mathrm{Ca}^{2+}$ stress. $(H, I){ }^{31} \mathrm{P}$ ssNMR analysis. Mitochondria $\left(70 \mathrm{mg} \mathrm{ml}^{-1}\right)$ were given acute treatment of SS-31 $(0.6 \mathrm{mM})$ or vehicle only followed by calcium treatment (up to $5 \mathrm{mM} \mathrm{CaCl}_{2}$ ). (H) Static wide-line ${ }^{31} \mathrm{P}$ NMR spectra of mitochondria subject to calcium stress $\left(5 \mathrm{mM} \mathrm{CaCl}_{2}\right)$ following treatment without (left) or with (right) SS-31. Spectral deconvolution yielded the indicated components (see Fig. S19), which were used to quantify the area ratios of L2:iso ( $n=3 \pm S D$ ) in panel I. (J) Respirometry analysis. Mitochondria $\left(1 \mathrm{mg} \mathrm{ml}^{-1}\right)$ were treated in the absence or presence of SS-31 $(54.4 \mu \mathrm{M})$ or $\mathrm{CaCl}_{2}$ $(1 \mathrm{mM})$ as indicated and measured for state 2 respiration $(n=10-12)$.

Fig. 8. SS peptide distribution and modulation of membrane surface electrostatics. $(A)$ Equilibrium binding of SS-31: (1) Polybasic peptide is drawn to bilayer surface under the force of the electric field originating from negative surface charge density. (2) Peptide interaction stabilized by polar contacts (basic side chains and headgroup phosphates) and hydrophobic interactions (burial of nonpolar side chains in acyl chain region). (B) Depiction of SS peptide equilibrium distribution at mitochondrial membranes showing mitochondrial cristae region, including the mitochondrial inner membrane (MIM), which delineates aqueous matrix and intracristal space (ICS). Topological positioning of the amphitropic protein cytochrome $c$ (cyt. $c$, PDB 1ycc) and $F_{0} F_{1}$ ATP synthase (CV dimer, PDB 6b8h) are shown for comparison. Dashed line represents region of $\sim 30 \AA$ (2-3 Debye lengths) from the membrane surface. Inset, representation of SS peptide distribution within the diffuse double layer. $(C)$ Working models for how regulation of mitochondrial surface charge may underpin the efficacy of SS peptides. (1 and 2) divalent cations and basic protein binding cause lipid demixing and sequestration of CL with implications for membrane integrity, lipid-protein interactions, and lipid peroxidation. The binding of SS peptides to CL-containing bilayers shifts the equilibrium binding of cations and basic proteins away from the membrane surface. (3) SS peptide binding alters physical properties of CL-containing bilayers. The ionized CL headgroup creates a strong local electric field (red) that alters lateral lipid interactions and creates a strong $\psi_{\mathrm{s}}$. Interfacial binding of SS31 reduces $\psi_{\mathrm{s}}$, thereby enhancing lipid packing and promoting local curvature. 


\section{References}

1. Gorman GS, et al. (2016) Mitochondrial diseases. Nat Rev Dis Primers 2:16080.

2. Nunnari J \& Suomalainen A (2012) Mitochondria: in sickness and in health. Cell 148(6):1145-1159.

3. Sorrentino V, Menzies KJ, \& Auwerx J (2018) Repairing Mitochondrial Dysfunction in Disease. Annu Rev Pharmacol Toxicol 58:353-389.

4. Szeto HH \& Liu S (2018) Cardiolipin-targeted peptides rejuvenate mitochondrial function, remodel mitochondria, and promote tissue regeneration during aging. Arch Biochem Biophys 660:137-148.

5. Szeto HH (2018) Stealth Peptides Target Cellular Powerhouses to Fight Rare and Common Age-Related Diseases. Protein Pept Lett.

6. Zhao K, Luo G, Giannelli S, \& Szeto HH (2005) Mitochondria-targeted peptide prevents mitochondrial depolarization and apoptosis induced by tert-butyl hydroperoxide in neuronal cell lines. Biochem Pharmacol 70(12):1796-1806.

7. Zhao K, Luo G, Zhao GM, Schiller PW, \& Szeto HH (2003) Transcellular transport of a highly polar 3+ net charge opioid tetrapeptide. J Pharmacol Exp Ther 304(1):425-432.

8. Zhao K, et al. (2004) Cell-permeable peptide antioxidants targeted to inner mitochondrial membrane inhibit mitochondrial swelling, oxidative cell death, and reperfusion injury. $J$ Biol Chem 279(33):34682-34690.

9. Winterbourn CC, Parsons-Mair HN, Gebicki S, Gebicki JM, \& Davies MJ (2004) Requirements for superoxide-dependent tyrosine hydroperoxide formation in peptides. Biochem J 381(Pt 1):241-248.

10. Birk AV, Chao WM, Bracken C, Warren JD, \& Szeto HH (2014) Targeting mitochondrial cardiolipin and the cytochrome c/cardiolipin complex to promote electron transport and optimize mitochondrial ATP synthesis. Br J Pharmacol 171(8):2017-2028.

11. Birk AV, Chao WM, Liu S, Soong Y, \& Szeto HH (2015) Disruption of cytochrome c heme coordination is responsible for mitochondrial injury during ischemia. Biochim Biophys Acta 1847(10):1075-1084.

12. Birk AV, et al. (2013) The mitochondrial-targeted compound SS-31 re-energizes ischemic mitochondria by interacting with cardiolipin. J Am Soc Nephrol 24(8):12501261.

13. Szeto $\mathrm{HH}$ (2014) First-in-class cardiolipin-protective compound as a therapeutic agent to restore mitochondrial bioenergetics. Br J Pharmacol 171(8):2029-2050.

14. Paradies G, Paradies V, De Benedictis V, Ruggiero FM, \& Petrosillo G (2014) Functional role of cardiolipin in mitochondrial bioenergetics. Biochim Biophys Acta 1837(4):408-417.

15. Musatov A \& Sedlak E (2017) Role of cardiolipin in stability of integral membrane proteins. Biochimie 142:102-111.

16. Planas-Iglesias J, et al. (2015) Cardiolipin Interactions with Proteins. Biophys J 109(6):1282-1294.

17. Mileykovskaya E \& Dowhan W (2014) Cardiolipin-dependent formation of mitochondrial respiratory supercomplexes. Chem Phys Lipids 179:42-48.

18. Alessandrini A \& Muscatello U (2009) AFM and FTIR spectroscopy investigation of the inverted hexagonal phase of cardiolipin. J Phys Chem B 113(11):3437-3444.

19. Lewis RN \& McElhaney RN (2009) The physicochemical properties of cardiolipin bilayers and cardiolipin-containing lipid membranes. Biochim Biophys Acta 1788(10):2069-2079.

20. Boyd KJ, Alder NN, \& May ER (2017) Buckling Under Pressure: Curvature-Based Lipid Segregation and Stability Modulation in Cardiolipin-Containing Bilayers. Langmuir 33(27):6937-6946. 
21. Ikon N \& Ryan RO (2017) Cardiolipin and mitochondrial cristae organization. Biochim Biophys Acta Biomembr 1859(6):1156-1163.

22. Schlame $M \&$ Greenberg ML (2017) Biosynthesis, remodeling and turnover of mitochondrial cardiolipin. Biochim Biophys Acta Mol Cell Biol Lipids 1862(1):3-7.

23. Lu YW \& Claypool SM (2015) Disorders of phospholipid metabolism: an emerging class of mitochondrial disease due to defects in nuclear genes. Front Genet 6:3.

24. Ikon N \& Ryan RO (2017) Barth Syndrome: Connecting Cardiolipin to Cardiomyopathy. Lipids 52(2):99-108.

25. Kopp Lugli A, Yost CS, \& Kindler CH (2009) Anaesthetic mechanisms: update on the challenge of unravelling the mystery of anaesthesia. Eur J Anaesthesiol 26(10):807-820.

26. Seelig J (1997) Titration calorimetry of lipid-peptide interactions. Biochim Biophys Acta 1331(1):103-116.

27. Boyd KJ, Alder NN, \& May ER (2018) Molecular Dynamics Analysis of Cardiolipin and Monolysocardiolipin on Bilayer Properties. Biophys J 114(9):2116-2127.

28. Pan J, et al. (2015) Structural and mechanical properties of cardiolipin lipid bilayers determined using neutron spin echo, small angle neutron and X-ray scattering, and molecular dynamics simulations. Soft Matter 11(1):130-138.

29. Walrant A, et al. (2012) Membrane interactions of two arginine-rich peptides with different cell internalization capacities. Biochim Biophys Acta 1818(7):1755-1763.

30. Witte K, Olausson BE, Walrant A, Alves ID, \& Vogel A (2013) Structure and dynamics of the two amphipathic arginine-rich peptides RW9 and RL9 in a lipid environment investigated by solid-state NMR and MD simulations. Biochim Biophys Acta 1828(2):824-833.

31. Swamy MJ \& Sankhala RS (2013) Probing the thermodynamics of protein-lipid interactions by isothermal titration calorimetry. Methods Mol Biol 974:37-53.

32. Anonymous (2002) Peptide-Lipid Interactions (Academic Press, San Diego).

33. Thorgeirsson TE, Russell CJ, King DS, \& Shin YK (1996) Direct determination of the membrane affinities of individual amino acids. Biochemistry 35(6):1803-1809.

34. Seelig J (2004) Thermodynamics of lipid-peptide interactions. Biochim Biophys Acta 1666(1-2):40-50.

35. McLaughlin S (1977) Electrostatic potentials at membrane-solution interfaces. Curr. Top. Membr. Transp. 9:71-144.

36. Oshima H (2010) Biophysical Chemistry of Interfaces (Wiley, Hoboken).

37. Slavik J (1982) Anilinonaphthalene sulfonate as a probe of membrane composition and function. Biochim Biophys Acta 694(1):1-25.

38. Willumeit R, et al. (2005) Structural rearrangement of model membranes by the peptide antibiotic NK-2. Biochim Biophys Acta 1669(2):125-134.

39. McLaughlin S, Mulrine N, Gresalfi T, Vaio G, \& McLaughlin A (1981) Adsorption of divalent cations to bilayer membranes containing phosphatidylserine. J Gen Physiol 77(4):445-473.

40. Kumar P, Kizhakkedathu JN, \& Straus SK (2018) Antimicrobial Peptides: Diversity, Mechanism of Action and Strategies to Improve the Activity and Biocompatibility In Vivo. Biomolecules 8(1).

41. Tyler AI, Law RV, \& Seddon JM (2015) X-ray diffraction of lipid model membranes. Methods Mol Biol 1232:199-225.

42. Seelig J (1978) 31P nuclear magnetic resonance and the head group structure of phospholipids in membranes. Biochim Biophys Acta 515(2):105-140.

43. Demchenko AP, Mely Y, Duportail G, \& Klymchenko AS (2009) Monitoring biophysical properties of lipid membranes by environment-sensitive fluorescent probes. Biophys $J$ 96(9):3461-3470. 
44. Cohen BE, et al. (2002) Probing protein electrostatics with a synthetic fluorescent amino acid. Science 296(5573):1700-1703.

45. Baile MG, et al. (2014) Unremodeled and remodeled cardiolipin are functionally indistinguishable in yeast. J Biol Chem 289(3):1768-1778.

46. Overall SA, Zhu S, Hanssen E, Separovic F, \& Sani MA (2019) In Situ Monitoring of Bacteria under Antimicrobial Stress Using (31)P Solid-State NMR. Int J Mol Sci 20(1).

47. Gasanov SE, et al. (2015) Naja naja oxiana Cobra Venom Cytotoxins CTI and CTII Disrupt Mitochondrial Membrane Integrity: Implications for Basic Three-Fingered Cytotoxins. PLoS One 10(6):e0129248.

48. Melcrova A, et al. (2016) The complex nature of calcium cation interactions with phospholipid bilayers. Sci Rep 6:38035.

49. Sani MA, Keech O, Gardestrom P, Dufourc EJ, \& Grobner G (2009) Magic-angle phosphorus NMR of functional mitochondria: in situ monitoring of lipid response under apoptotic-like stress. FASEB J 23(9):2872-2878.

50. Shewan A, Eastburn DJ, \& Mostov K (2011) Phosphoinositides in cell architecture. Cold Spring Harb Perspect Biol 3(8):a004796.

51. Bigay J \& Antonny B (2012) Curvature, lipid packing, and electrostatics of membrane organelles: defining cellular territories in determining specificity. Dev Cell 23(5):886-895.

52. Jakovcic S, Swift HH, Gross NJ, \& Rabinowitz M (1978) Biochemical and stereological analysis of rat liver mitochondria in different thyroid states. J Cell Biol 77(3):887-901.

53. Krebs JJ, Hauser H, \& Carafoli E (1979) Asymmetric distribution of phospholipids in the inner membrane of beef heart mitochondria. J Biol Chem 254(12):5308-5316.

54. Hwang MS, et al. (2014) Mitochondrial $\mathrm{Ca}(2+)$ influx targets cardiolipin to disintegrate respiratory chain complex II for cell death induction. Cell Death Differ 21(11):1733-1745.

55. Granatiero V, De Stefani D, \& Rizzuto R (2017) Mitochondrial Calcium Handling in Physiology and Disease. Adv Exp Med Biol 982:25-47.

56. Kagan VE, et al. (2009) Cytochrome c/cardiolipin relations in mitochondria: a kiss of death. Free Radic Biol Med 46(11):1439-1453.

57. Camilleri A, et al. (2013) Mitochondrial membrane permeabilisation by amyloid aggregates and protection by polyphenols. Biochim Biophys Acta 1828(11):2532-2543.

58. Sciacca MFM, Tempra C, Scollo F, Milardi D, \& La Rosa C (2018) Amyloid growth and membrane damage: Current themes and emerging perspectives from theory and experiments on Abeta and hIAPP. Biochim Biophys Acta Biomembr.

59. Manczak M, et al. (2010) Mitochondria-targeted antioxidants protect against amyloidbeta toxicity in Alzheimer's disease neurons. J Alzheimers Dis 20 Suppl 2:S609-631.

60. Reddy PH, Manczak M, \& Kandimalla R (2017) Mitochondria-targeted small molecule SS31: a potential candidate for the treatment of Alzheimer's disease. Hum Mol Genet 26(8):1483-1496.

61. Dahlberg M (2007) Polymorphic phase behavior of cardiolipin derivatives studied by coarse-grained molecular dynamics. J Phys Chem B 111(25):7194-7200.

62. Khalifat N, Fournier JB, Angelova MI, \& Puff N (2011) Lipid packing variations induced by $\mathrm{pH}$ in cardiolipin-containing bilayers: the driving force for the cristae-like shape instability. Biochim Biophys Acta 1808(11):2724-2733.

63. Lewis RN \& McElhaney RN (2000) Surface charge markedly attenuates the nonlamellar phase-forming propensities of lipid bilayer membranes: calorimetric and (31)P-nuclear magnetic resonance studies of mixtures of cationic, anionic, and zwitterionic lipids. Biophys J 79(3):1455-1464.

64. $\mathrm{Xu} \mathrm{Y,} \mathrm{et} \mathrm{al.} \mathrm{(2016)} \mathrm{Loss} \mathrm{of} \mathrm{protein} \mathrm{association} \mathrm{causes} \mathrm{cardiolipin} \mathrm{degradation} \mathrm{in} \mathrm{Barth}$ syndrome. Nat Chem Biol 12(8):641-647. 
A<smiles>Cc1cc(O)cc(C)c1C[C@H](NC(=O)[C@H]([NH3+])CCCNC(N)=[NH2+])C(=O)N[C@@H](CCCC[NH3+])C(=O)N[C@@H](Cc1ccccc1)C(N)=O</smiles>

B<smiles>NC(=O)[C@H](CCCC[NH3+])NC(=O)[C@H](Cc1ccccc1)NC(=O)[C@H](CCCNC(N)=[18O])NC(=O)[C@H]([NH3+])Cc1ccccc1</smiles>

C<smiles>Cc1cc(O)ccc1C[C@H](NC(=O)[C@H](CCCCN)NC(=O)C(N)N)C(=O)N[C@@H](CC(=O)c1ccc2cc(N(C)C)ccc2c1)C(N)=O</smiles>

Fig. 1. SS peptide chemical structures. (A) SS-31 (D-Arg-2'6'-dimethylTyr-Lys-Phe- $\mathrm{NH}_{2}$ ). (B) SS-20 (Phe-DArg-Phe-Lys- $\mathrm{NH}_{2}$ ). (C) [ald]SS-31, SS-31 with an aladan moiety (red) in place of Phe. 
bioRxiv preprint doi: https://doi.org/10.1101/735001; this version posted August 14,2019 . The copyright holder for this preprint (which was not

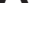
certified by peer review) is the aubor/funder. All rights reserved. No reuse allowed without permission $\mathbf{C}$
ptipid titration peptide titration
[lipid] $(\mu \mathrm{M})\left[\begin{array}{ccc}\bullet 25 & 50 & 75 \\ -100 & \bullet 125 & \end{array}\right]$ $[S S-31](\mu \mathrm{M})\left[\begin{array}{cc}2.5 & \bullet 5 \\ 10 & \bullet 15\end{array}\right]$
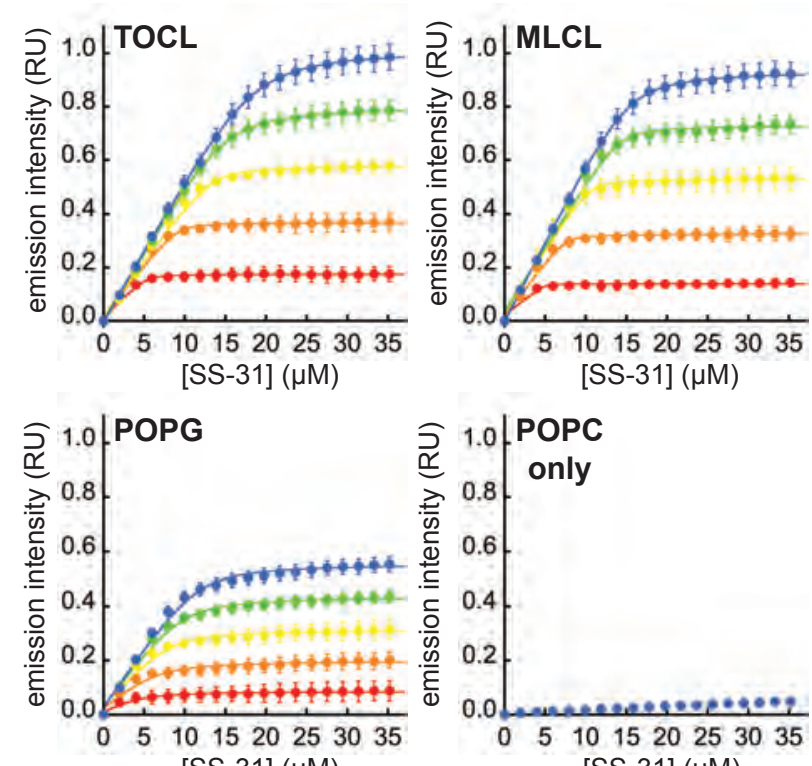

[SS-31] $(\mu \mathrm{M})$
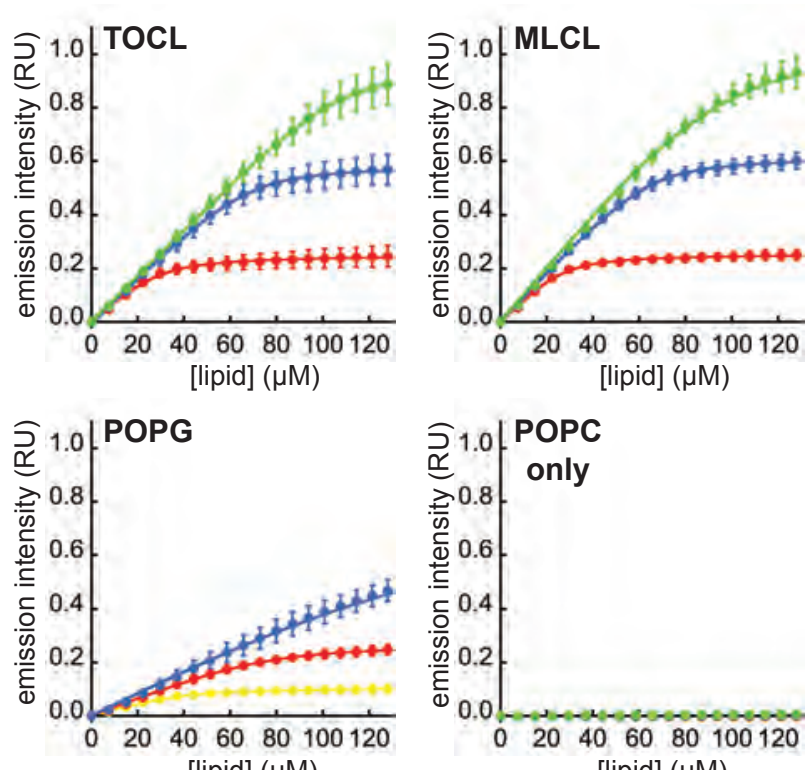

[lipid] $(\mu \mathrm{M})$

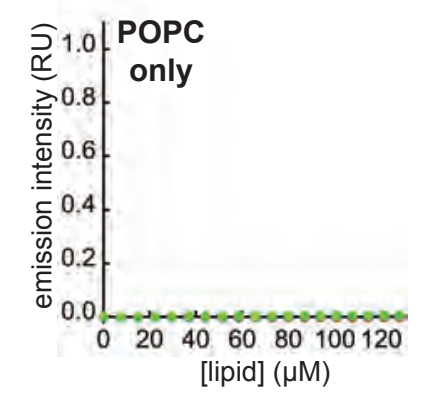

$[\bullet T O C L \quad \bullet M L C L \quad \bullet P O P G]$
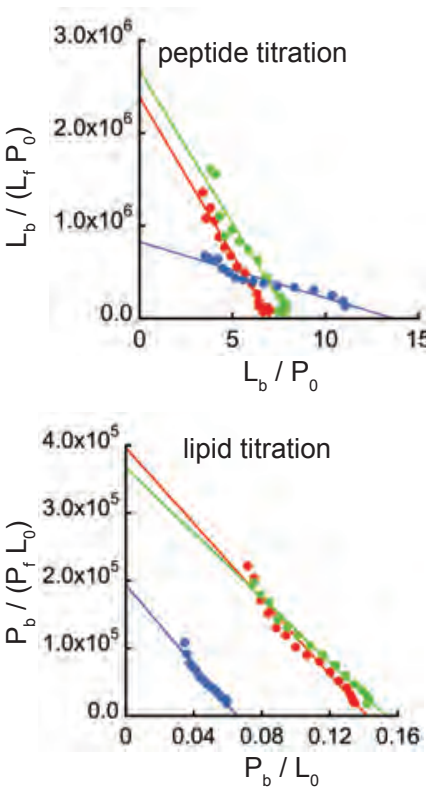

E

$\left[\begin{array}{lll}\square O C L & \mathrm{MLCL} & \square \mathrm{POPG}\end{array}\right]$

ITC measurements
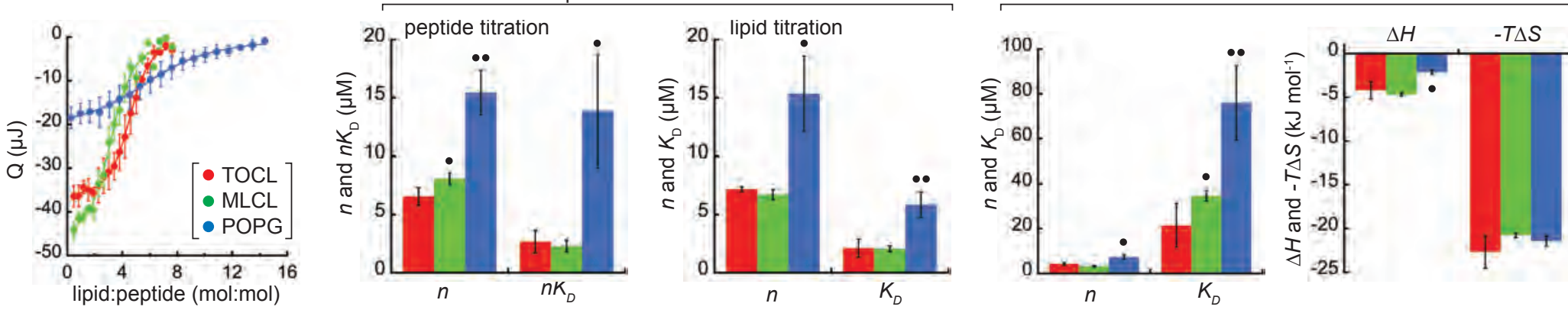

Fig. 2. SS-31 binding isotherms. (A-C) Fluorescence-based measurements. $(A)$ Peptide titrations. Liposomes containing anionic lipid (20 mol\% TOCL, MLCL, or POPG) or POPC only at the lipid concentrations indicated were titrated with SS-31 (4 nmol increments). Values shown are means ( $n=3 \pm S D$ ) and lines show data fits based on Eq. S2. (B) Lipid titrations. Peptides at the concentrations indicated were titrated with liposomes (30 nmol lipid increments). Values shown are means ( $n=3 \pm S D$ ) and lines show data fits based on Eq. S3. (C) Scatchard analyses. Titration data with liposomes containing $20 \mathrm{~mol} \%$ TOCL, MLCL, and POPG shown as Scatchard plots. Upper panel, peptide titrations (125 $\mu \mathrm{M}$ lipid) fit according to Eq. S6; lower panel, lipid titrations (10 $\mu \mathrm{M}$ SS-31, TOCL and MLCL; $5 \mu \mathrm{M}$ SS-31, POPG) fit according to Eq. S8. (D) ITC analyses. Wiseman plot showing blank-subtracted average integrated heats $(n=3 \pm S D)$ as a function of lipid:SS-31 molar ratio for LUVs containing TOCL, MLCL, and POPG. (E) Summary of binding parameter data. Statistical differences within each dataset determined relative to TOCL-containing membranes: $P<0.05 ; \bullet P<0.01$. 
bioRxiv preprint doi: https://doi.org/10.1101/735001; this version posted August 14,2019 . The copyright holder for this preprint (which was not A added $[0 \quad 10$ crtified by peer review) is the author/funder. All rights reserved 0 No reuse allowed without permission.

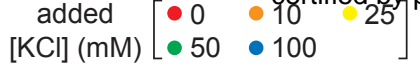

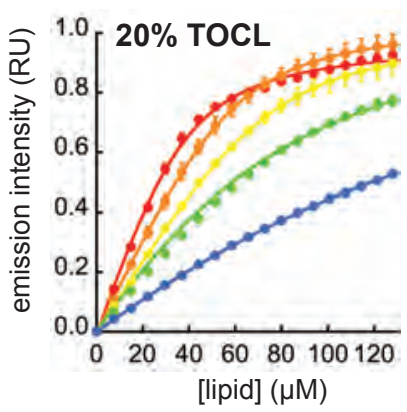

B
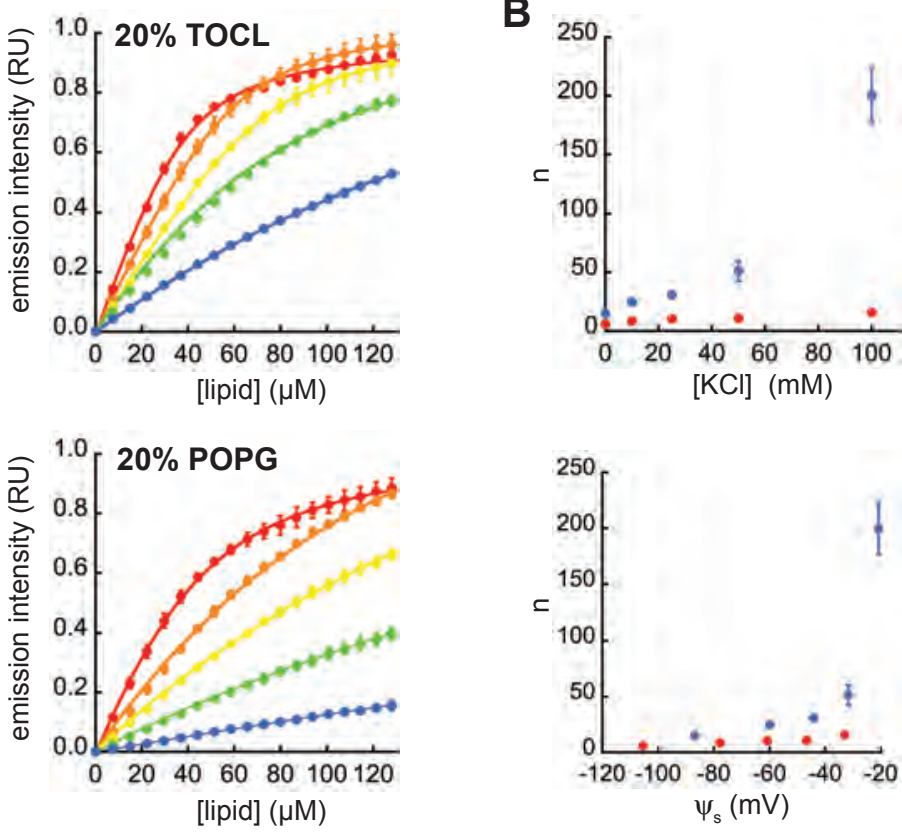

LUV type $[\bullet 20 \%$ TOCL $\bullet 20 \%$ POPG $]$

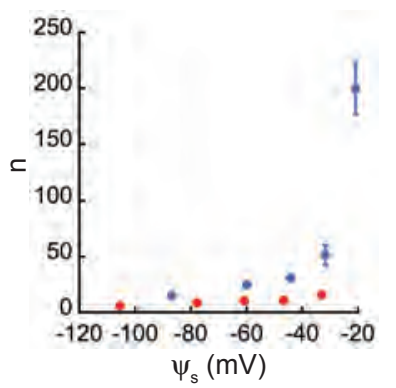

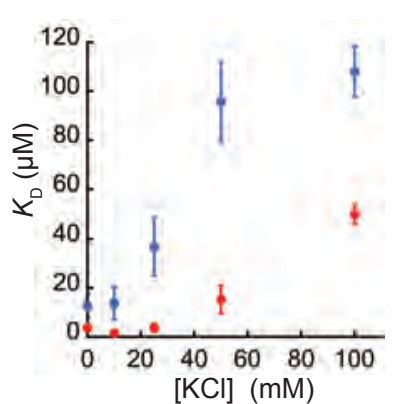

D

C


Fig. 3. SS-31 binding and surface electrostatics. (A) Binding isotherms. Peptide was titrated with LUVs of the indicated composition (20\% TOCL, [SS-31] $=7.5 \mu \mathrm{M}$ and 20\% POPG, [SS-31] = $3.8 \mu \mathrm{M})$ at $30 \mathrm{nmol}$ lipid increments in the presence of different concentrations of added KCl. Values shown are means $(n=3 \pm S D)$ and lines show data fits based on Eq. S3. Because isotherms at higher [KCl] did not reach saturation, curves within each membrane set were fit globally assuming a common saturation point. $(B)$ Equilibrium binding parameters $n$ and $K_{\mathrm{D}}$ at different ionic strengths as a function of [KCl] (upper panels) and as a function of $\psi_{\mathrm{s}}$, calculated from GCS formalism, Eq. S10-S12 (lower panels) based on binding isotherms of panel A. (C,D) Effect of SS-31 binding on surface charge. Measurements of $(C)$ zeta potential $(\zeta)$ and $(D)$ ANS fluorescence shown as a function of [SS-31]:[Lip] $]^{\text {eff }}$ for membranes containing 20\% TOCL and 20\% POPG as indicated (values are means, $n=3 \pm S D$ ). 
A bioRxiv preprint doi: https://doi.org/10.1101/735001; this version posted August 14,2019 . The copyright holder for this preprint (which was not

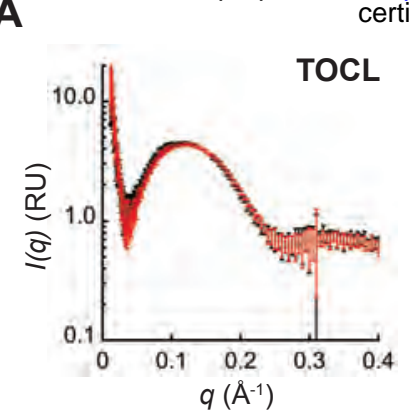

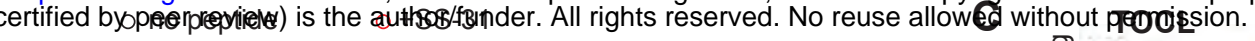

B
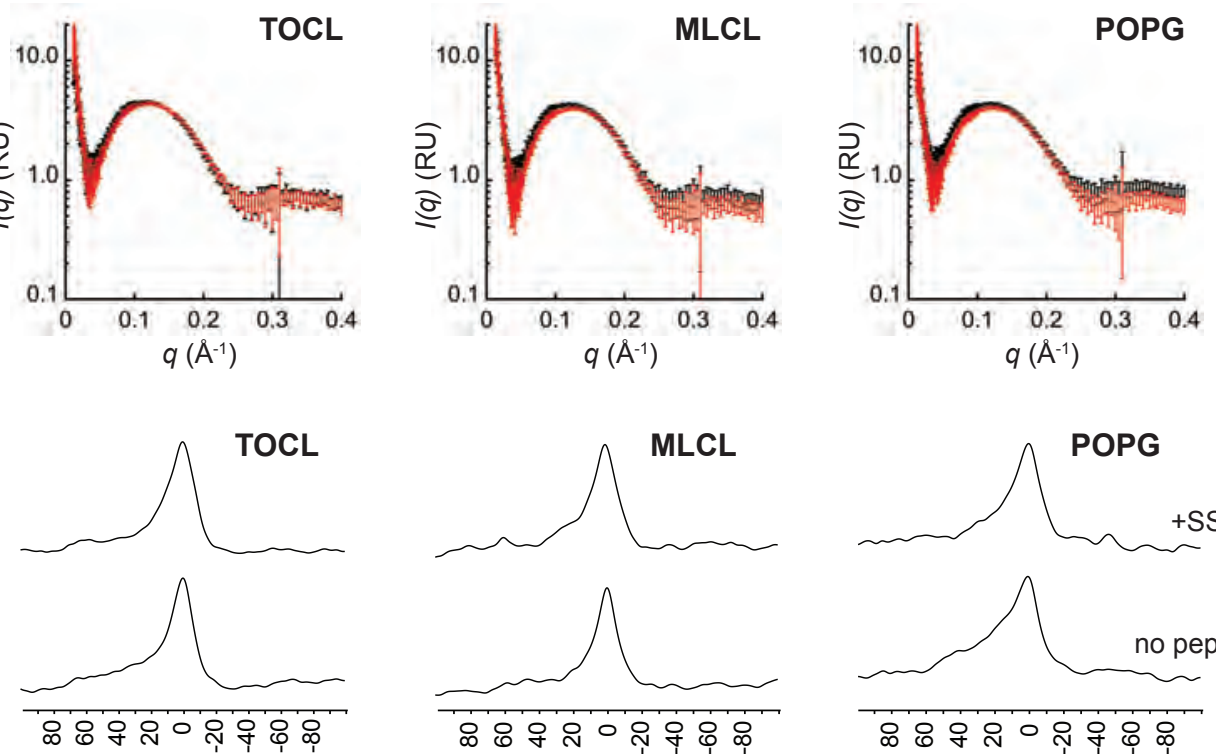

f1 (ppm)
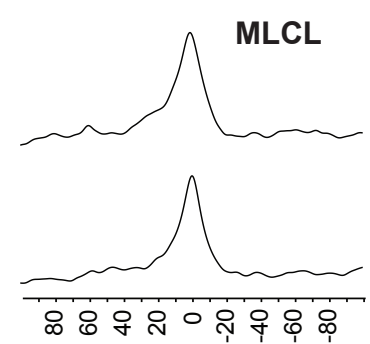

f1 (ppm)

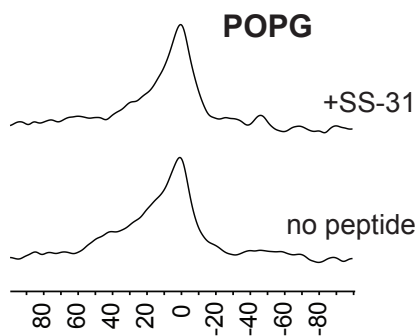

$\mathrm{f1}(\mathrm{ppm})$
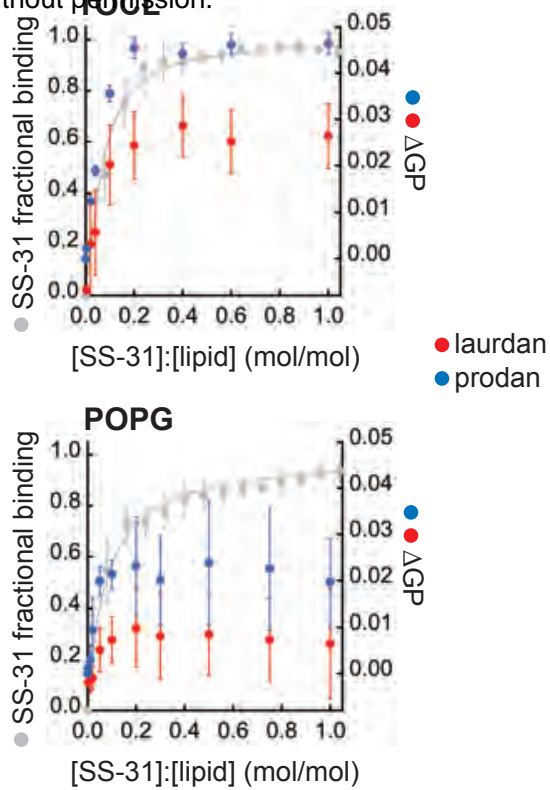

Fig. 4. Effects of SS-31 on structural properties of model membranes. $(A, B)$ SS-31 does not cause major structural perturbation of model membranes. LUVs composed of $20 \%$ anionic lipid (TOCL, MLCL, or POPG) in POPC background were measured in the absence or presence of peptide ([P]: $\left.[L]^{\text {eff }}=1: 5\right)$ as indicated. $(A)$ Synchrotron SAXS measurements showing background-subtracted scattering density profiles for LUVs in the absence (black) or presence (red) of SS-31. (B) ${ }^{31} \mathrm{P}$ solid state NMR spectra of LUVs in the absence (lower spectra) or presence (upper spectra) of SS-31. (C) SS-31 alters lipid packing interactions. Changes in laurdan/prodan GP values $(\Delta G P)(n=3 \pm S D)$ with increasing [SS-31] for LUVs containing $20 \%$ TOCL or POPG in POPC background as indicated. Data and fits in grey show SS-31 fractional saturation (calculated from Fig. $2 \mathrm{~A}, 25 \mu \mathrm{M}$ lipid). 
A

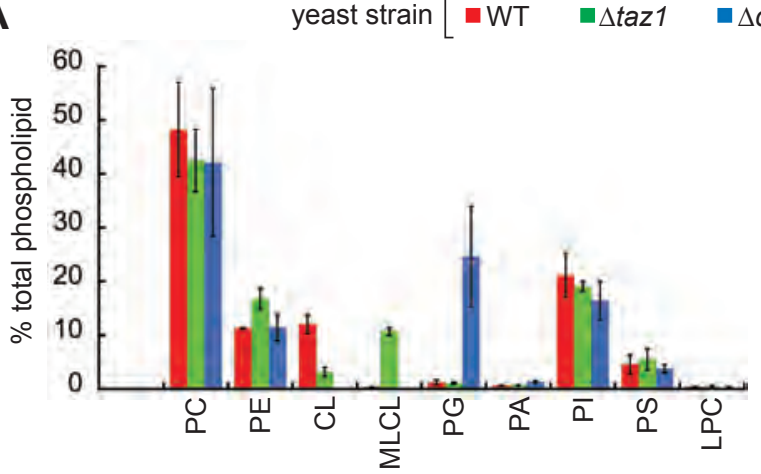

phospholipid species



B

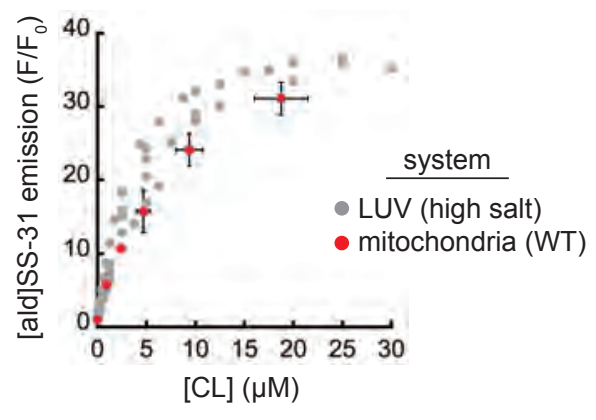

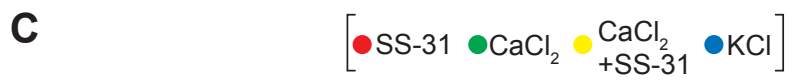

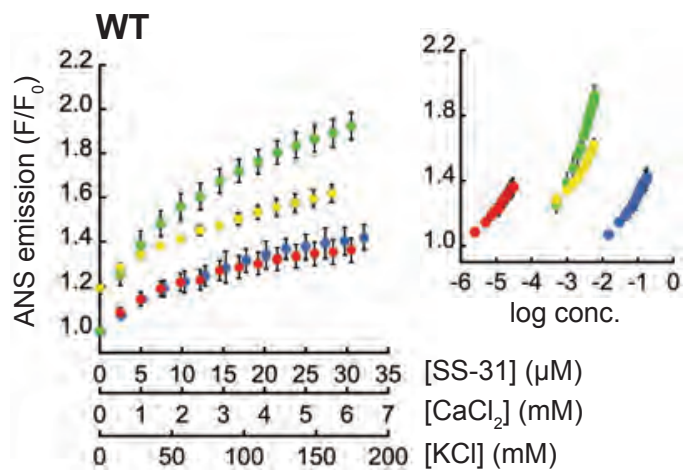

Fig. 6. Interaction of SS-31 with isolated mitochondria. $(A)$ [ald]SS-31 binding with differing phospholipid composition. Left, Percentages of total phospholipid of each species (PC, phosphatidylcholine; PE, phosphatidylethanolamine; CL, cardiolipin; MLCL, monolyso-CL; PG, phosphatidylglycerol; PA, phosphatidic acid; PI, phosphatidylinositol; PS, phosphatidylserine; LPC, lyso-phosphatidylcholine) in mitochondria from WT, $\Delta$ taz1, and $\Delta c r d 1$ strains measured by shotgun lipidomics. Right, binding of $1 \mu \mathrm{M}$ [ald]SS-31 with mitochondria isolated from WT, $\Delta t a z 1$, and $\Delta c r d 1$ yeast strains quantified as the mean fractional increase $\left(F / F_{0}\right)$ in aladan emission ( $\left.n=3 \pm S D\right)$ with increasing mitochondria concentration for fully energized mitochondria. Inset, relative binding of [ald]SS-31 to mitochondria $\left(0.2 \mathrm{mg} \mathrm{ml}^{-1}\right)$ in the presence or absence of $1 \mu \mathrm{M}$ valinomycin ("val") as indicated. (B) Comparison of [ald]SS-31 binding to mitochondria and model membranes. [ald]SS-31 binding is shown as a function of CL concentration for: (i) LUVs of defined TOCL composition under high salt conditions (gray, Fig. S15) and (ii) mitochondria isolated from yeast WT strain (red, Figs. S16 and 6A). Calculation of [CL] for mitochondrial samples was based on lipidomics analyses of Fig. 6A. (C) Effects of SS-31 and salt cations on mitochondrial surface potential. Fractional change in ANS emission is shown for titration mitoplasts from WT (left) and $\Delta c r d 1$ (right) yeast with SS-31, $\mathrm{CaCl}_{2}$, or $\mathrm{KCl}$ at the indicated concentrations $\left(n=3 \pm S D\right.$ ). For WT samples, $\mathrm{CaCl}_{2}$ titrations were also conducted following the addition of 20 $\mu \mathrm{M}$ SS-31 ("CaCl${ }_{2}+\mathrm{SS}-31$ "). Insets, ANS emission data plotted as a function of log concentration for all three titrants. 
A

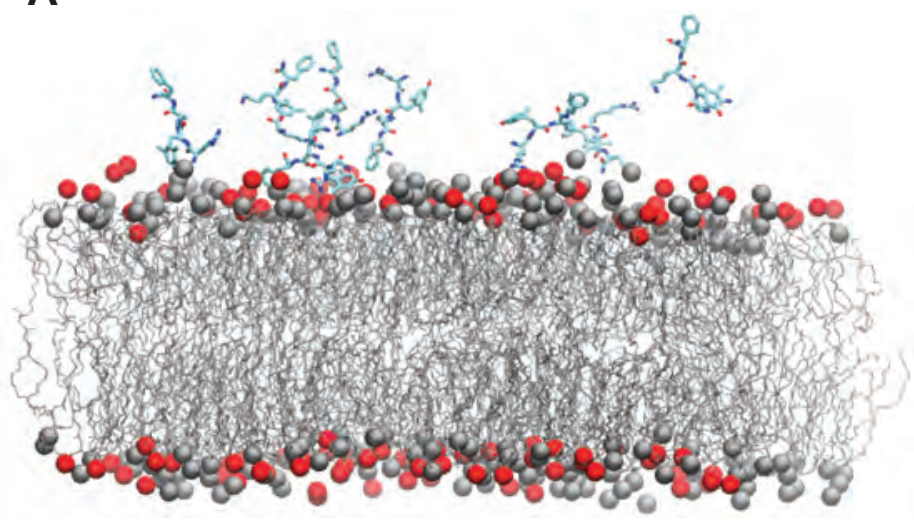

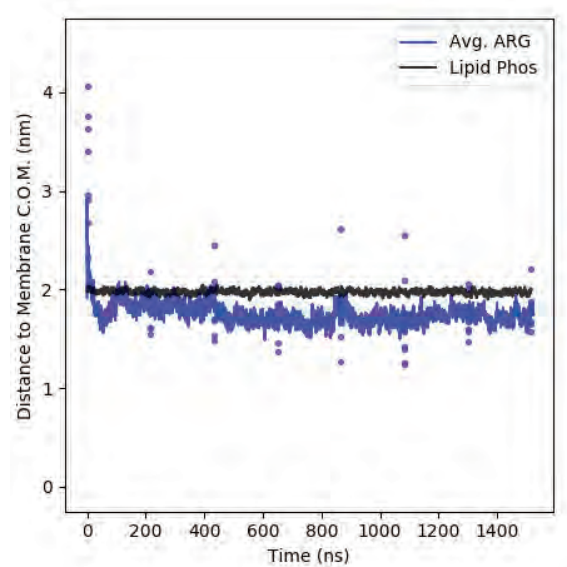

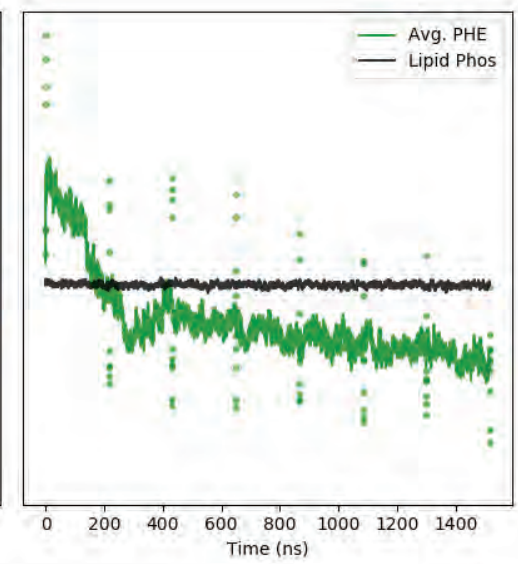

C peptide approach

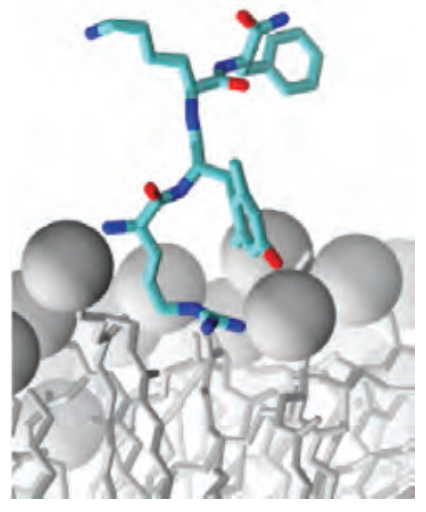

peptide burial

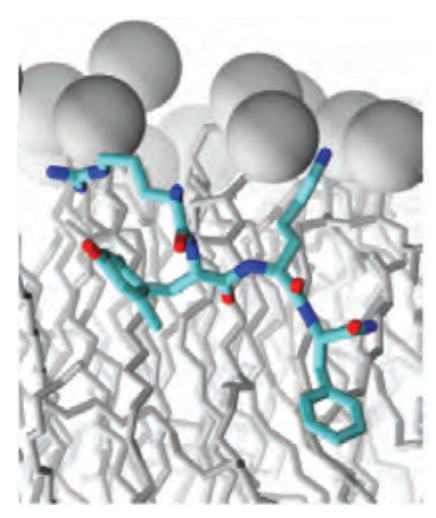

D
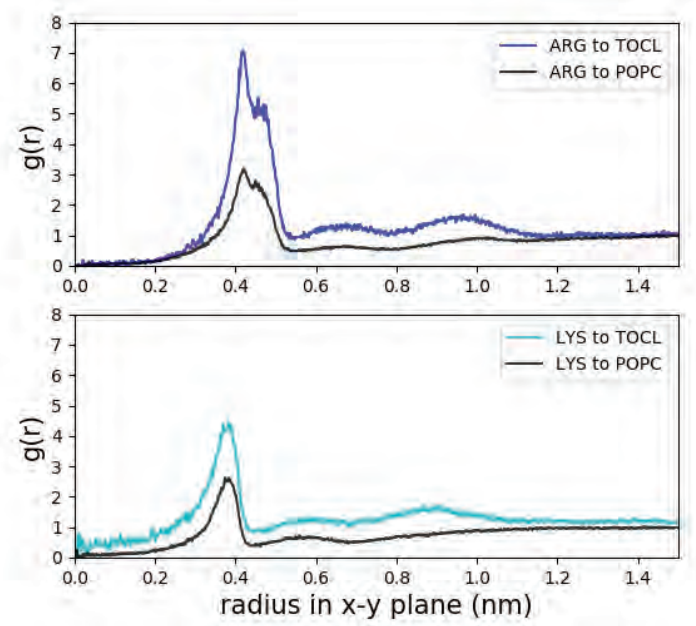

Fig. 5. Molecular dynamics simulations of SS-31 bilayer interactions. (A) Snapshot of a typical MD simulation with SS-31 peptides in the aqueous phase relative to the upper leaflet of a $20 \%$ TOCL bilayer. Peptides are shown in licorice representation (cyan, carbon; blue, nitrogen; red, oxygen), lipid acyl chains are in wireframe, and lipid phosphates are shown as van der Waals spheres for POPC (gray) and TOCL (red). (B) Temporal evolution of SS-31 Arg (blue) and Phe (green) side chain positions relative to the membrane center of mass (C.O.M.). Black lines show the average position of all phosphates in the upper leaflet, colored dots show $z$ coordinates $\left(z^{P O S}\right)$ of individual side chains, and colored lines represent average $z^{P O S}$ values. $(C)$ Orientation of SS-31 bound to a lipid bilayer consisting of $20 \mathrm{~mol} \% \mathrm{TOCL}$ in a POPC background in the peptide approach (left) and buried (right) states. Peptide representation as in $(A)$, with all lipid phosphates shown as gray van der Waals spheres. $(D)$ Lipid distributions around basic SS-31 residues. Lateral ( $x-y$ plane) radial distribution profiles from Arg and Lys side chains to lipid headgroup phosphate atoms of TOCL or POPC as indicated. 

certified by peer review) ig the author/funder. All rights reserved. No reuse allowed without permission.

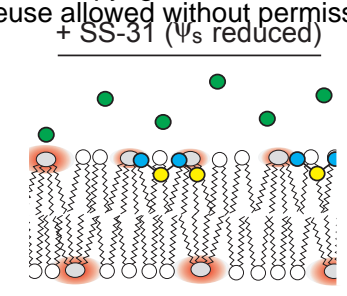

B $\quad$ ss-3

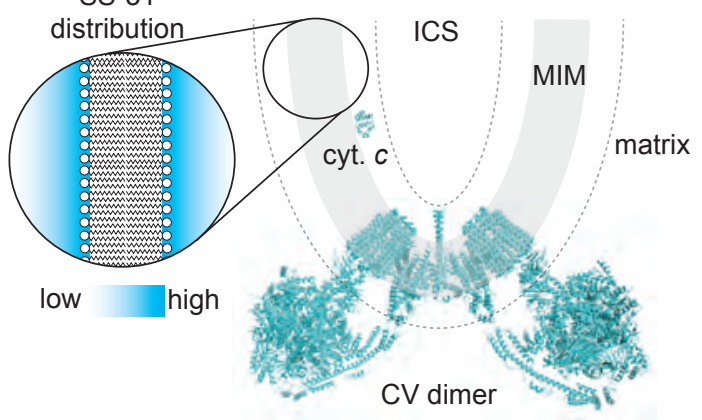

binding of polybasic proteins
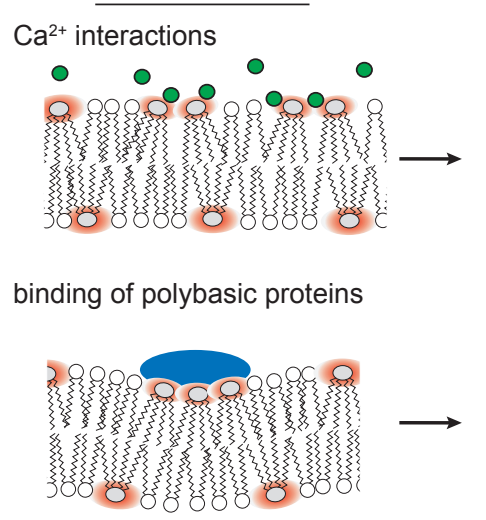

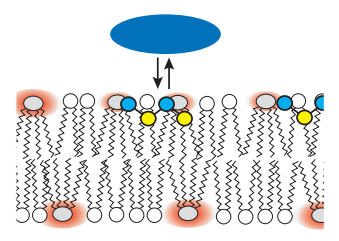
potentiating curvature/packing

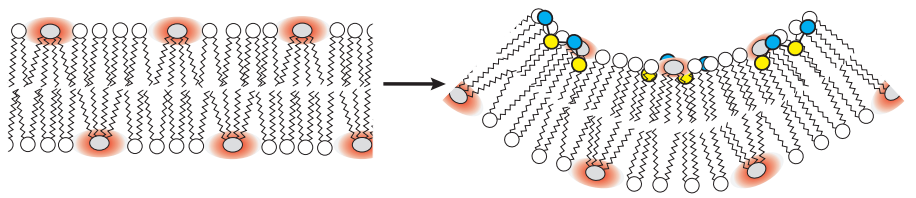

solution
electrolytes $\left[\begin{array}{c}\circ \text { monovalent cation } \\ \circ \text { monovalant anion } \\ \circ \mathrm{Ca}^{2+}\end{array}\right.$

Fig. 8. SS peptide distribution and modulation of membrane surface electrostatics. $(A)$ Equilibrium binding of SS-31: (1) Polybasic peptide is drawn to bilayer surface under the force of the electric field originating from negative surface charge density. (2) Peptide interaction stabilized by polar contacts (basic side chains and headgroup phosphates) and hydrophobic interactions (burial of nonpolar side chains in acyl chain region). (B) Depiction of SS peptide equilibrium distribution at mitochondrial membranes showing mitochondrial cristae region, including the mitochondrial inner membrane (MIM), which delineates aqueous matrix and intracristal space (ICS). Topological positioning of the amphitropic protein cytochrome $c$ (cyt. $c$, PDB 1ycc) and $F_{0} F_{1}$ ATP synthase (CV dimer, PDB 6b8h) are shown for comparison. Dashed line represents region of $\sim 30 \AA$ (2-3 Debye lengths) from the membrane surface. Inset, representation of SS peptide distribution within the diffuse double layer. (C) Working models for how regulation of mitochondrial surface charge may underpin the efficacy of SS peptides. ( 1 and 2) divalent cations and basic protein binding cause lipid demixing and sequestration of $\mathrm{CL}$ with implications for membrane integrity, lipid-protein interactions, and lipid peroxidation. The binding of SS peptides to CL-containing bilayers shifts the equilibrium binding of cations and basic proteins away from the membrane surface. (3) SS peptide binding alters physical properties of CL-containing bilayers. The ionized CL headgroup creates a strong local electric field (red) that alters lateral lipid interactions and creates a strong $\psi_{\mathrm{s}}$. Interfacial binding of SS-31 reduces $\psi_{s}$, thereby enhancing lipid packing and promoting local curvature. 